\title{
Distinct Dendritic Spine and Nuclear Phases of Calcineurin Activation after Exposure to Amyloid- $\beta$ Revealed by a Novel Fluorescence Resonance Energy Transfer Assay
}

\author{
Hai-Yan Wu, ${ }^{1}$ Eloise Hudry, ${ }^{1}$ Tadafumi Hashimoto, ${ }^{1}$ Kengo Uemura, ${ }^{1}$ Zhan-Yun Fan, ${ }^{1}$ Oksana Berezovska, ${ }^{1}$ \\ Cynthia L. Grosskreutz, ${ }^{2}$ Brian J. Bacskai, ${ }^{1}$ and Bradley T. Hyman ${ }^{1}$ \\ ${ }^{1}$ Department of Neurology/Alzheimer's Disease Research Laboratory, Massachusetts General Hospital, Charlestown, Massachusetts 02129, and \\ ${ }^{2}$ Department of Ophthalmology, Massachusetts Eye and Ear Infirmary, Boston, Massachusetts 02114
}

Calcineurin $(\mathrm{CaN})$ activation is critically involved in the regulation of spine morphology in response to oligomeric amyloid- $\beta(\mathrm{A} \beta)$ as well as in synaptic plasticity in normal memory, but no existing techniques can monitor the spatiotemporal pattern of CaN activity. Here, we use a spectral fluorescence resonance energy transfer approach to monitor CaN activation dynamics in real time with subcellular resolution. When oligomeric $\mathrm{A} \beta$ derived from $\mathrm{Tg} 2576$ murine transgenic neurons or human $\mathrm{AD}$ brains were applied to wild-type murine primary cortical neurons, we observe a dynamic progression of $\mathrm{CaN}$ activation within minutes, first in dendritic spines, and then in the cytoplasm and, in hours, in the nucleus. CaN activation in spines leads to rapid but reversible morphological changes in spines and in postsynaptic proteins; longer exposure leads to NFAT (nuclear factor of activated T-cells) translocation to the nucleus and frank spine loss. These results provide a framework for understanding the role of calcineurin in synaptic alterations associated with AD pathogenesis.

\section{Introduction}

Calcineurin $(\mathrm{CaN})$ is a calcium-dependent serine/threonine phosphatase that regulates ion channels, cytoskeletal proteins, and transcription factors (Klee et al., 1979; Halpain et al., 1998; Berridge et al., 2000; Winder and Sweatt, 2001). CaN plays fundamental roles in neuronal development, regulating axonal cone outgrowth (Lautermilch and Spitzer, 2000), dendritic complexity, and synaptic function (Schwartz et al., 2009). Alteration of $\mathrm{CaN}$ activity was also reported in neuropathological contexts such as Parkinson's disease (Martin et al., 2012) and Alzheimer's disease (Liu et al., 2005).

Several biochemical methods exist to monitor CaN activity (Blumenthal et al., 1986; Enz et al., 1994; Sellar et al., 2006), but they suffer from a lack of anatomical precision and specificity. Translocation of dephosphorylated nuclear factor of activated T-cells (NFAT) to the nucleus is also used to assess CaN activity, but this indirect method requires stimulation for hours and is sensitive to the balance between NFAT dephosphorylation (by CaN) and phosphorylation [by GSK3 $\beta$ (Newman and Zhang, 2008)]. CaN has a wide spectrum of substrates in neurons such as cofilin, AMPA recep-

\footnotetext{
Received Jan. 16, 2012; revised Feb. 25, 2012; accepted Feb. 28, 2012

Author contributions: H.-Y.W., K.U., O.B., B.J.B., and B.T.H. designed research; H.-Y.W., E.H., and T.H. performed research; K.U., Z.-Y.F., O.B., C.L.G., and B.J.B. contributed unpublished reagents/analytic tools; H.-Y.W., E.H., and T.H. analyzed data; H.-Y.W., E.H., and B.T.H. wrote the paper.

This work was supported by National Institutes of Health Grants AG08487, EB000768, EY13399, and 1R01AG041507-01. We thank Dr. Jeffery D. Molkentin (Cincinnati Children's Hospital, Cincinnati, OH) for providing CaN constructs. We thank Daniel Joyner for primary neuron preparation.

The authors declare no competing financial interests.

Correspondence should be addressed to Dr. Bradley T. Hyman, MassGeneral Institute for Neurodegenerative Disease, 114 16th Street, Charlestown, MA 02129. E-mail: bhyman@partners.org.

DOI:10.1523/JNEUROSCI.0227-12.2012

Copyright $\odot 2012$ the authors $\quad 0270-6474 / 12 / 325298-12 \$ 15.00 / 0$
}

tors, BAD (Bcl2 antagonist of cell death), and NFAT (Springer et al., 2000; Tavalin et al., 2002; Morishita et al., 2005; Wang et al., 2005; Wu et al., 2010). These downstream targets are localized in different cellular compartments, but current approaches cannot address the dynamics of $\mathrm{CaN}$ activation at a subcellular level, and are not suitable to temporally follow the changes in CaN activity.

We postulated that fluorescence resonance energy transfer (FRET) assays, increasingly used to detect molecular interactions in cells with spatiotemporal resolution (Stryer, 1978; Selvin, 2000; Jares-Erijman and Jovin, 2003), might be capable of measuring $\mathrm{CaN}$ activation. CaN phosphatase activity is physiologically regulated by calcium, $\mathrm{CaN} B$ subunit (CaNB) and calmodulin (CaM) (Klee et al., 1979; Aramburu et al., 2000). Under basal conditions, $\mathrm{CaN}$ is inactivated by a regulatory inhibitory domain. After an intracellular calcium influx, CaM and CaNB bind to their target sites on CaNA, triggering a conformational change leading to the displacement of the autoinhibitory domain from the enzymatic site (Wang et al., 1989; Klee et al., 1998). Based on CaNA interactions with $\mathrm{CaNB}$ and $\mathrm{CaM}$, we developed a FRET assay to measure CaNA-CaNB or CaNA-CaM interaction or conformational change using either endogenous proteins or fluorescent tagged molecules.

We show that FRET assays can efficiently monitor CaN activation in different subcellular compartments. Imaging two-color fluorescence for CaNA-CaM or CaNA-CaNB pairs allowed us to interrogate the activation status of $\mathrm{CaN}$ in single neurons. We observed low levels of activity in the resting state. After a brief exposure to oligomeric amyloid- $\beta(\mathrm{A} \beta)$ prepared from Tg2576 conditioned media or purified from human $\mathrm{AD}$ cortex, we detected a rapid $\mathrm{CaN}$ activation in spines with a later activation in the cytoplasm and, ultimately, in the nucleus. Washout of the oligomeric $\mathrm{A} \beta$ prevented 
A
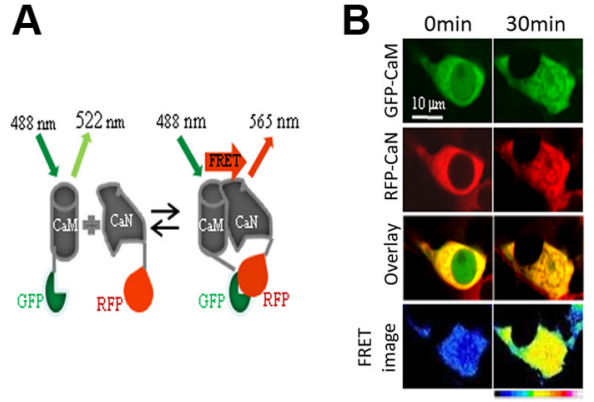

D RFP-CAn signal GFP-CAM signal
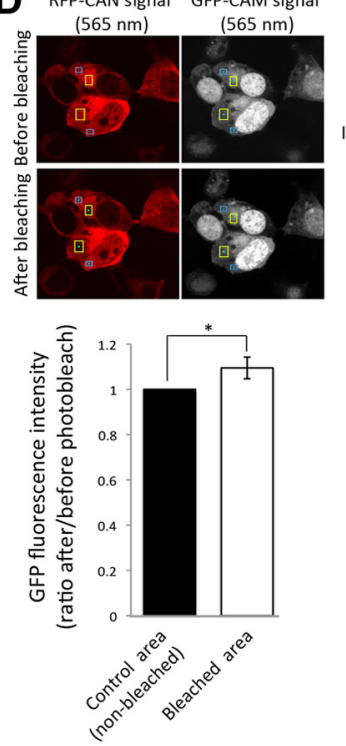

E
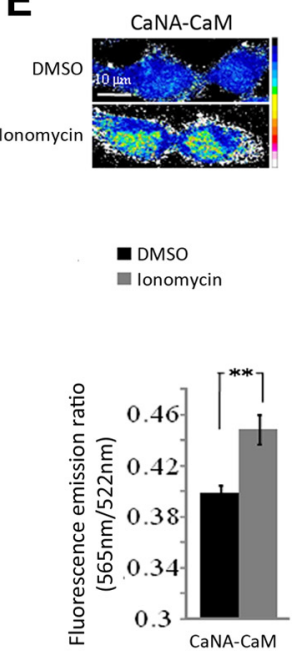

C

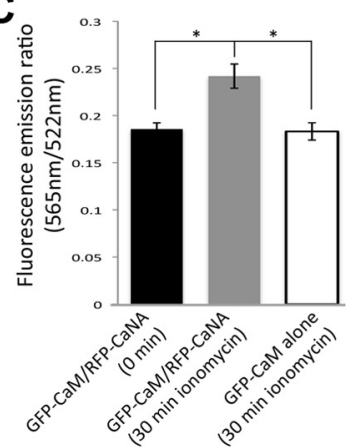

$\mathbf{F}$

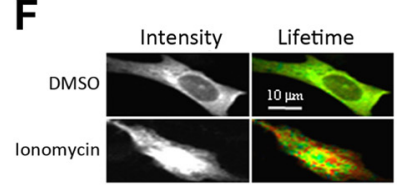

DMSO

lonomycin

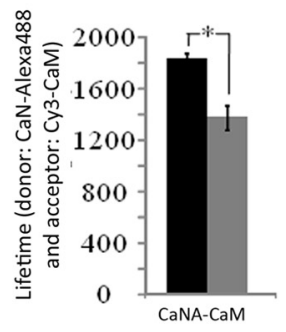

Figure 1. FRET efficiency between CaNA-CaM or CaN-CaNB represents CaN activation in HEK cells. $\boldsymbol{A}$, FRET strategy that was used for detection of CaN-CaM interaction. B, Images of HEK cells transiently transfected with GFP-CaM and RFP-CaNA for $24 \mathrm{~h}$. Cells were excited at GFP-specific excitation wavelength $(488 \mathrm{~nm})$. The FRET ratios were calculated by reporting emission intensity of RFP, measured at $565 \mathrm{~nm}$, to the GFP emission peak measured at $522 \mathrm{~nm}$. The pseudocolored ratio images (FRET images) represent the emission changes before (time, $0 \mathrm{~min}$ ) and $30 \mathrm{~min}$ after the addition of $2 \mu \mathrm{m}$ ionomycin. Cells treated with ionomycin for 30 min showed higher FRET compared with 0 min. The color scale (bottom right) indicates that a shift toward the red end of the spectrum corresponds to higher FRET efficiency. C, Quantitative analysis of normalized FRET ratio in HEK cells transiently transfected with GFP-CaM and RFP-CaNA for $24 \mathrm{~h}$ and treated with $2 \mu \mathrm{m}$ ionomycin for $30 \mathrm{~min}$. No change is detected when the donor alone (GFP-CaM) was transfected. D, To confirm the existence of a FRET signal between GFP-CaM and RFP-CaN, a photobleach experiment was performed. HEK cells were transfected with both GFP-CaM and RFP-CaNA and treated for 30 min with ionomycin. After fixation, the RFP fluorescent signal of a small cytoplasmic region of interest was photobleached with $100 \%$ excitation light at $543 \mathrm{~nm}$ for $5 \mathrm{~min}$ (top panel, yellow boxes), and the intensity of the GFP signal was measured before and after bleaching. As a control, the GFP intensity was quantified in nonbleached areas as well (top panel, blue boxes). An increase of the GFP-CaM emission intensity was observed in areas where the RFP signal was photobleached, but no change was detected in control areas. $\boldsymbol{E}$, Endogenous change in FRET signal was quantified by staining the cells for endogenous CaNA-Alexa 488 and endogenous CaM or CaNB-Cy3. The top panel represents pseudocolored ratio images (FRET images) observed in HEK cells treated with ionomycin or DMSO as a vehicle control for $30 \mathrm{~min}$. The bottom panel shows quantitative analysis of normalized spectral ratio $\left(n \geq 60\right.$ cells; ${ }^{*} p<$ $\left.0.05 ;{ }^{* *} p<0.001\right) . F$, FLIM analysis of the interaction between CaNA and CaM in HEK cells treated with $2 \mu$ m ionomycin for 30 min. After treatment, cells were stained with endogenous CaNA-Alexa 488 and endogenous CaM-Cy3. The top panel, on the left, shows intensity images of Alexa 488 fluorescence reflecting the pattern of endogenous CaNA. The top panel, on the right, shows pseudocolored FLIM images. The bottom panel shows quantitative FLIM analysis of in HEK cells treated with DMSO and ionomycin. Cells stained with CaNA-Alexa 488 were used as a negative control to establish Alexa 488 lifetime in the absence of an acceptor fluorophore (Cy3) (data not shown). Lifetime is in picoseconds; ${ }^{*} p<0.05 ; n \geq 25$ cells. Data represent mean \pm SEM.

progression to the cytoplasm or nucleus. The rapid activation of $\mathrm{CaN}$ in spines alters spine morphology and reduces postsynaptic proteins including F-actin and GluR1-containing AMPA receptors. The distinct spatial and temporal distribution of CaN activation supports the idea that metabolic processes in different cellular compartments are selectively targeted by $\mathrm{CaN}$ during $\mathrm{A} \beta$ neurotoxicity.

\section{Materials and Methods}

Primary neuronal cultures. All animals were used in accordance with animal protocols approved by the Institutional Animal Care and Use Committee. Neuronal cultures were generated using transgenic embryos from either sex expressing human mutated APP gene [Tg2576 line: transgenic mice overexpressing the 695 aa isoform of human Alzheimer $\beta$-amyloid precursor protein containing the double Swedish mutation K670N, M671L with a hamster prion protein gene promoter in $\mathrm{B} 6$; $\mathrm{SJL} \mathrm{F}_{2}$ mice (Hsiao et al., 1996)]. Primary neuronal cultures were derived from cerebral cortex of embryonic day 15-19 Tg2576 mice (Charles River Laboratories), as described previously with modifications (Wu et al., 2004). Briefly, cortices were dissected, gently minced, trypsinized $\left(0.027 \%, 37^{\circ} \mathrm{C} ; 5 \% \mathrm{CO}_{2}\right.$ for 15 $\min )$, and then washed with $1 \times$ HBSS. Neurons were seeded to a density of $4 \times 10^{5}$ viable cells/35 mm culture dishes previously coated with poly-D-lysine $(100 \mu \mathrm{g} / \mathrm{ml})$ for at least $1 \mathrm{~h}$ at $37^{\circ} \mathrm{C}$. Cultures were maintained at $37^{\circ} \mathrm{C}$ with $5 \% \mathrm{CO}_{2}$, supplemented with Neurobasal medium with 2\% B27 nutrient, $2 \mathrm{~mm}$ L-glutamine, $100 \mathrm{U} / \mathrm{ml}$ penicillin, and $100 \mu \mathrm{g} / \mathrm{ml}$ streptomycin. The cultures were used within $28 \mathrm{~d}$ in vitro (DIV). To maintain elevated levels of extracellular $\mathrm{A} \beta$, media were not changed. To identify the genotype of the animals, we used PCR on DNA extracted from sample tail taken after dissection of the cerebral cortex.

Plasmid construction. The cDNA of human CaM (NM-001743) was cloned into peGFP-C1 vector (Clontech) using BamH1 restriction site. RFP and mouse CaN were cloned into peGFP-N1 vector (Clontech) using Kpn1/ Age1 (RFP) and Age1/Not1(mCaN), respectively. Authenticity of all constructs was confirmed by sequencing.

Immunostaining of HEK cells and cortical primary neurons. To detect endogenous CaNA, CaNB and CaM, HEK cells or primary neurons were fixed with $4 \%$ paraformaldehyde for 15 min and were permeabilized with $0.5 \%$ Triton X-100 in PBS for 5 min. After blocking with 3\% bovine serum albumin (BSA) at $37^{\circ} \mathrm{C}$ for $1 \mathrm{~h}$, cells were incubated with primary antibodies: anti-CaNA (1:200; Calbiochem), anti-CaM antibody (1:200; Calbiochem), or anti-CaNB antibody (1:200; Millipore) overnight at $4^{\circ} \mathrm{C}$. Cells were then washed four times and incubated with secondary antibodies conjugated to either cyanine 3 (Cy3) (1:500; Jackson ImmunoResearch) or Alexa 488 (1:500; Invitrogen). Each antibody was diluted in PBS containing $1.5 \%$ BSA.

Surface AMPA receptors were measured as described below. In brief, 21 DIV primary neurons treated with wild-type or TgCM for 60 min were fixed in $4 \%$ paraformaldehyde (20 min; room temperature), but not permeabilized. Following an incubation with $3 \%$ bovine serum albumin $(1 \mathrm{~h})$ to block nonspecific staining, neurons were incubated with anti-NT-GluR1 (1:500; Millipore) overnight at $4^{\circ} \mathrm{C}$. After three washes, neurons were incubated with Cy3-conjugated secondary antibody (Jackson ImmunoResearch) for $2 \mathrm{~h}$ at room temperature. After washing in PBS three 
times, the coverslips were mounted on slides with Vectashield mounting medium. For staining of F-actin, neurons were incubated with Oregon Green 488 phalloidin ( $1 \mathrm{U} / \mathrm{ml}$; Invitrogen) at room temperature for $20 \mathrm{~min}$. Fluorescent images were captured using an LSM 510 Carl Zeiss microscope with a $25 \times$ or a $63 \times$ water-immersion objective lens [numerical aperture (NA), 0.8 and 1.2, respectively]. All images were taken at $512 \times 512$ pixel resolution and quantified with the ImageJ software. To define dendritic clusters, a single threshold was chosen manually so that clusters corresponded to puncta of twofold to threefold greater intensity than the diffuse fluorescence on the dendritic shaft. On each coverslip, the cluster density, size, and fluorescence intensity of four to six GFP-positive neurons (two to three dendritic segments of $\sim 50 \mu \mathrm{m} /$ neuron) were compared. Quantitative analyses were conducted without knowledge of the experimental conditions.

Quantitative spectral FRET assays. Spectral FRET was performed as described previously (Uemura et al., 2009). Briefly, HEK293 cells in $35 \mathrm{~mm}$ glass bottom dishes were either transfected with CaNA-RFP and CaM-GFP fused constructs, or immunolabeled for endogenous CaNA, CaNB, and CaM. Cells were imaged $24 \mathrm{~h}$ after the transfection. Spectral imaging was performed on a Zeiss LSM510 microscope equipped with a $63 \times$ water-immersion objective lens (NA, 1.2) and with $37^{\circ} \mathrm{C}$ heating chamber containing $5 \% \mathrm{CO}_{2}$. The wavelengths used for FRET imaging were $488 \mathrm{~nm}$ (excitation of GFP), separated by $488 / 563$ dichroic mirror, and emitted fluorescence was detected by seven channels of the Metadetector with $21.4 \mathrm{~nm}$ spectral bandwidth for each channel that was set to detect fluorescence in the 502$651 \mathrm{~nm}$ wavelength range. When FRET occurs, the energy is transferred from GFP (donor) to RFP (acceptor); thus, the GFP emission peak weakens, and the fluorescence intensity of RFP increases. Analogous measures were made for Alexa 488 (donor) and Cy3 (acceptor) in immunofluorescent-based assays (Knowles et al., 1999). We used the ratio of 565 to $522 \mathrm{~nm}$ as readout of the FRET efficiency, which reflects the relative proximity between each two fluorophores. The ratio value was calculated by dividing the average pixel fluorescence intensity at $565 \mathrm{~nm}$ by the intensity at $522 \mathrm{~nm}$. The ratiometric image was produced by dividing the intensity of the image from the $565 \mathrm{~nm}$ spectral window by the intensity from the $522 \mathrm{~nm}$ window after background subtraction, using ImageJ. For representation of the data, a look-up table was applied by mapping image values to color scale ( 16 colors) resulting in pseudocolor image. We used 10 and $25 \times$ objectives for the average-per-cell analysis, and $63 \times$ objective for studying subcellular distribution of $\mathrm{CaN}$ activation.

A photobleach experiment was performed as a control for FRET. HEK cells were transfected with GFP-CaM and RFP-CaNA using the same amount of each plasmid. After $2 \mathrm{~d}$, ionomycin (or DMSO) was applied

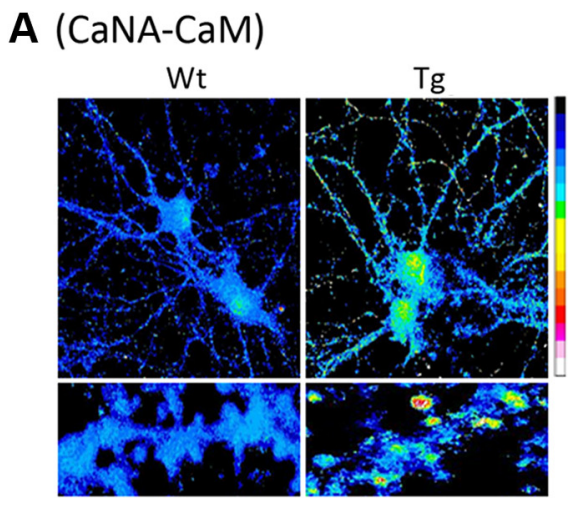

B

$$
\text { C (CaNA-CaM) }
$$
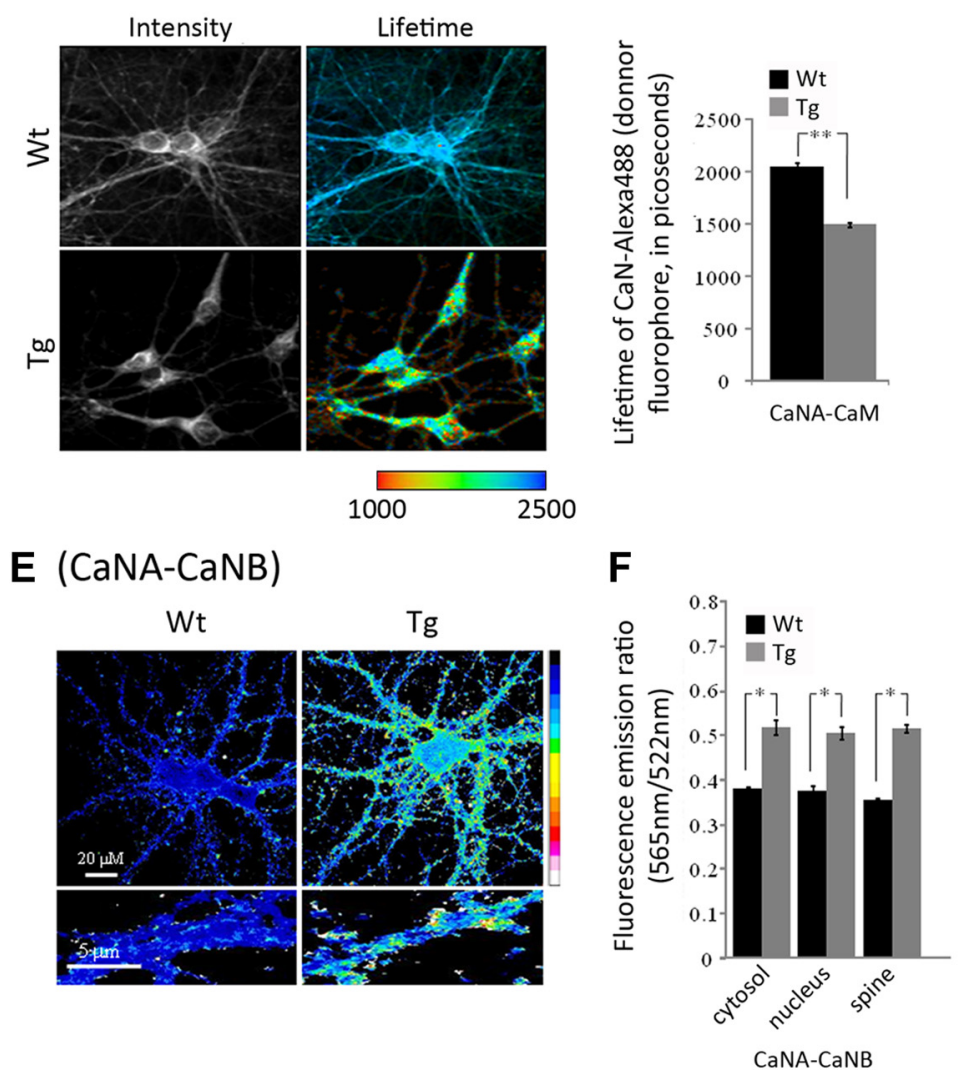

Figure 2. Comparison of CaN activation at subcellular level by spectral FRET assay in an in vitro model of AD. $A$, Representative FRET images of endogenous CaNA-Alexa 488/CaM-Cy3 observed in neurons prepared from wild-type and Tg2576 embryos. B, The bar graph represents the quantitative analysis of normalized spectral ratio (intensity ratio of $565-522 \mathrm{~nm}$ ) from different neuronal compartments in both Wt and Tg neurons. Neurons from Tg2576 culture had significantly higher FRET between CaNA-Alexa 488 and CaM-Cy3 compared with neurons from wild-type cultures. C, FLIM analysis of the interaction of CaNA-CaM in Wt and Tg2576 neurons. Wt or Tg2576 neurons at 21 DIV were stained with endogenous CaNA-Alexa 488 (donor) and endogenous CaM-Cy3 (acceptor). The left panel shows intensity images of Alexa 488 fluorescence reflecting the pattern of endogenous CaNA. The right panel shows pseudocolored FLIM images. $D$, Quantitative FLIM analysis of Wt or Tg2576 neurons. Cells stained with CaNA-Alexa 488 were used as a negative control to determine Alexa 488 lifetime in the absence of an acceptor fluorophore (Cy3) (data not shown). Lifetime is in picoseconds. $\boldsymbol{E}$, Representative FRET images of endogenous CaNA-Alexa 488/CaNB-Cy3 from neurons of wild-type and Tg2576 culture. $\boldsymbol{F}$, The bar graph represents the quantitative analysis of normalized spectral ratio (intensity ratio of $565-522 \mathrm{~nm}$ ) from different neuron compartments in both wild-type and Tg2576 neurons. Neurons from Tg2576 culture had significantly higher FRET between CaNA-Alexa 488 and CaNB-Cy3 in the cytosol, nuclei, and spines compared with that of neurons from wild-type cultures. $n \geq 50$ cells; ${ }^{*} p<0.05$; ${ }^{* *} p<0.001$. Data represent mean \pm SEM.

for $30 \mathrm{~min}$ to induce $\mathrm{CaN}$ activation and cells were then fixed with $4 \%$ paraformaldehyde for $15 \mathrm{~min}$. First, the intensity of the GFP signal was measured in a small cytoplasmic region of interest (ROI) of the cell. To bleach the acceptor signal, the selected ROI was scanned with 100\% laser at $543 \mathrm{~nm}$ for $5 \mathrm{~min}$. The photobleach efficiency was confirmed by the 
loss of any fluorescent signal at $565 \mathrm{~nm}$, and the intensity of the GFP fluorophore in the same area was measured again. To determine whether the photobleach of the RFP signal leads to an increase of the donor fluorescence intensity, the ratio of the GFP signal after and before photobleach was calculated. This experiment showed enhanced GFP signal within the acceptor photobleached area, consistent with dequenching of the donor fluorophore and FRET. As an internal control, the GFP signal of another ROI where the RFP signal was not photobleached was unchanged.

Fluorescence lifetime imaging. Fluorescence lifetime imaging (FLIM) assay was performed in HEK cells and cultured neurons that were previously stained for endogenous CaNA and CaM, as previously described. Briefly, cells were imaged on a Zeiss LCM510 microscope with a $63 \times$ water-immersion objective lens (NA, 1.2) and a Chameleon pulsed laser was used to excite Alexa 488 donor fluorophore (two-photon excitation wavelength at $840 \mathrm{~nm}$ ). Becker \& Hickl FLIM hardware and software (Becker \& Hickl) were used to acquire the donor lifetime information. Data analysis was performed using SPC Image (Becker \& Hickl), in which the donor fluorophore lifetimes are determined by fitting the data to one (negative control) or two (experimental conditions) exponential decay curves. The donor lifetime information was color coded and displayed as pseudocolored images (Berezovska et al., 2005).

Analysis of spine density and spine morphology. Cultured neurons (7 DIV) were transfected with green fluorescent protein and examined at 21 DIV using a LSM 510 Zeiss confocal microscope (excitation laser at 488 $\mathrm{nm}$ ). Each image consisted of a $z$ stack of pictures taken at a depth interval of $0.8 \mu \mathrm{m}$ and then projected into one image. The densities of filopodia and spines were measured separately. For the analysis of density, filopodia or spines were defined as dendritic protrusions of $0.5-3 \mu \mathrm{m}$ in length, with (spine) or without (filopodia) a head. Density was calculated by dividing the filopodia or spine number by the measured length of the correspondent neurite they were connected to. Filopodia and spines were specifically evaluated in the distal dendrites of the neurons. In each experiment, $>200$ spines in $>12$ neurons were examined for each treatment and three experiments were performed. For each condition, measurements of spine densities were averaged per neuron; the means from multiple neurons were then averaged to obtain the mean \pm SEM for the population of neurons.

Size exclusion chromatography and quantification of $A \beta$. As described previously (Hashimoto et al., 2002), $1 \mathrm{~g}$ of cortical gray matter from frontal lobe of $\mathrm{AD}$ patient brain or non-demented control brain (from either sex) was homogenized in $5 \mathrm{vol}$ of TBSI (Tris-buffered saline with protease inhibitor mixture; Roche) with 25 strokes on a mechanical Dounce homogenizer and centrifuged at $260,000 \times g$ for $20 \mathrm{~min}$ at $4^{\circ} \mathrm{C}$. The supernatant was used as TBS-soluble fraction. A volume of $750 \mu \mathrm{l}$ of TBS-soluble fraction of brain was separated by size exclusion chromatography (SEC) on Superdex 75 10/300 GL column (GE Healthcare) in $50 \mathrm{~mm}$ ammonium acetate, $\mathrm{pH} 8.5$, with AKTA purifier 10 (GE Healthcare) (Townsend et al., 2006). To quantify $A \beta 40$ and $A \beta 42$, eluted fractions were diluted and subjected to BNT77/BA27 for A $\beta 40$, or BNT77/ BC05 for A $\beta 42$ two-site ELISAs (Wako Chemicals).

Statistical analyses. Data are reported as mean \pm SEM, and statistical significance was defined at $p<0.05$. We made comparisons between groups by one- or two-way ANOVA, followed by post hoc Bonferroni's test for comparison among means.

\section{Results}

Characterization of $\mathrm{Ca}^{2+}$-induced CaNA-CaM or CaNACaNB interaction by spectral FRET in HEK cells

We first examined the interaction of CaNA and CaM in HEK cells, in which CaN activation can be induced by application of a $\mathrm{Ca}^{2+}$ ionophore (Bittinger et al., 2004; Park et al., 2006). If CaN can be activated by transient increases in intracellular $\mathrm{Ca}^{2+}$ following ionomycin application, the interaction of CaNA with CaM should take place because activation of $\mathrm{CaN}$ requires binding of CaM (Wang et al., 1989; Klee et al., 1998). We first examined the interaction of CaNA-CaM in the presence of elevated intracellular $\mathrm{Ca}^{2+}$ in living HEK cells expressing GFP-CaM and

\section{A (CaNA-CaM)}

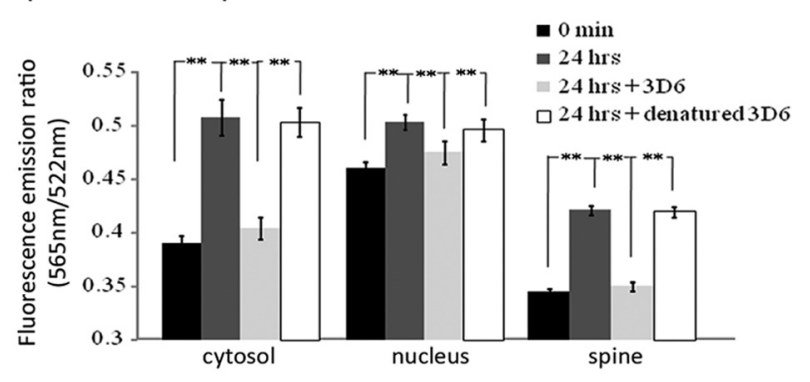

\section{B (CaNA-CaNB)}

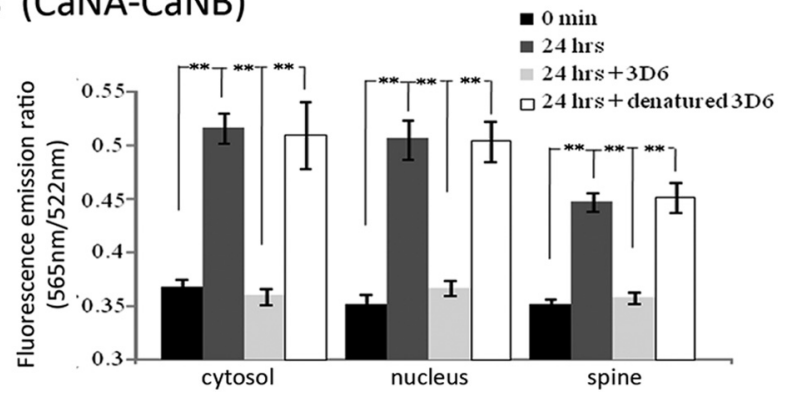

Figure 3. FRET assay reports sustained CaN activation in wild-type neurons treated with $A \beta$-containing $\operatorname{TgCM} . \boldsymbol{A}, \boldsymbol{B}$, Quantitative analysis of the normalized spectral ratio (intensity ratio of 565-522 nm) shows the extent of FRET from wild-type neurons (21 DIV) treated with A $\beta$ containing Tg2576 conditioned media for $24 \mathrm{~h}$. Using either endogenous CaNA-CaM $(\boldsymbol{A})$ and endogenous CaNA-CaNB assay $(\boldsymbol{B})$, we observed that $\mathrm{TgCM}$ treatment leads to higher FRET ratio in cortical neurons compared with wild-type conditioned media. This increase was prevented by immunodepletion of the $\operatorname{TgCM}$ with the anti-A $\beta$ antibody, 3D6, but no effect could be detected when 3D6 was previously denatured. $n \geq 60$ cells; ${ }^{* *} p<0.001$. Data represent mean \pm SEM.

RFP-CaNA (Fig. 1 $A, B$ ). To measure spectral FRET, cells were excited with $488 \mathrm{~nm}$ laser (donor), and the emission of the acceptor RFP was recorded at $565 \mathrm{~nm}$ (emission peak of RFP; Fig. $1 A$ ). If CaNA and CaM physically interact (within 10-50 $\AA$ ), an excitation light at $488 \mathrm{~nm}$ should result in a significantly enhanced RFP emission at $565 \mathrm{~nm}$, as a result of the energy transfer from the GFP-CaM donor to RFP-CaNA acceptor. We determined the ratio of fluorescence intensity between 565 to $522 \mathrm{~nm}$ as readout for the spectral FRET efficiency: higher 565/522 ratio indicates higher FRET efficiency, and hence increased interaction. Figure $1 B$ shows images of HEK cell expressing GFP-CaM and RFPCaNA before and after stimulation with $2 \mu \mathrm{M}$ ionomycin, in which the RFP/GFP emission ratio is represented as pseudocolored images. In cells coexpressing GFP-CaM and RFP-CaNA, high FRET was observed after ionomycin treatment, whereas lower but detectable FRET was observed before ionomycin treatment (Fig. $1 B, C$ ). Cells expressing the fluorescent donor CAMGFP alone showed no change in the 565/522 ratio after stimulation (Fig. 1C), indicating that the change in fluorescence observed at $565 \mathrm{~nm}$ emission wavelength is due to the energy transfer from the GFP fluorophore to the RFP fluorophore fused to their respective proteins. As an additional technical control to ensure that the 565/522 ratio change reflected FRET, a photobleach experiment of the acceptor fluorophore was performed. After treatment with ionomycin, the RFP-CaNA was photobleached by scanning a small ROI with $100 \%$ laser at $543 \mathrm{~nm}$ for $5 \mathrm{~min}$. An increased intensity of the donor fluorophore due to donor dequenching (Fig. 1D) was detected in the same ROI, thus confirming that any increase in the 565/522 ratio is associated with a transfer of energy from the donor to the acceptor fluoro- 
A

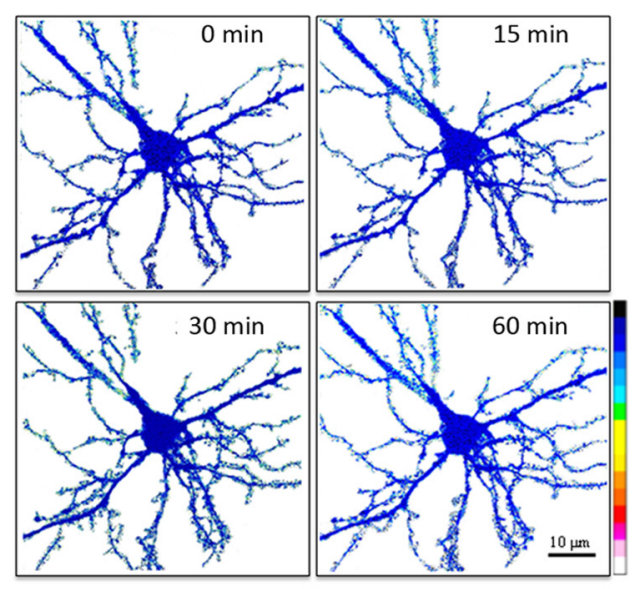

C
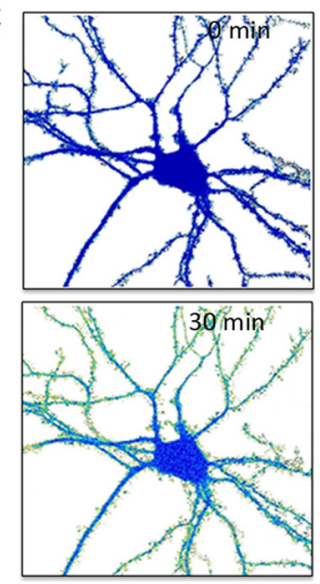
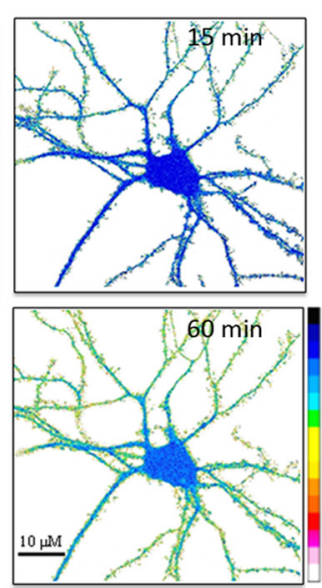

B
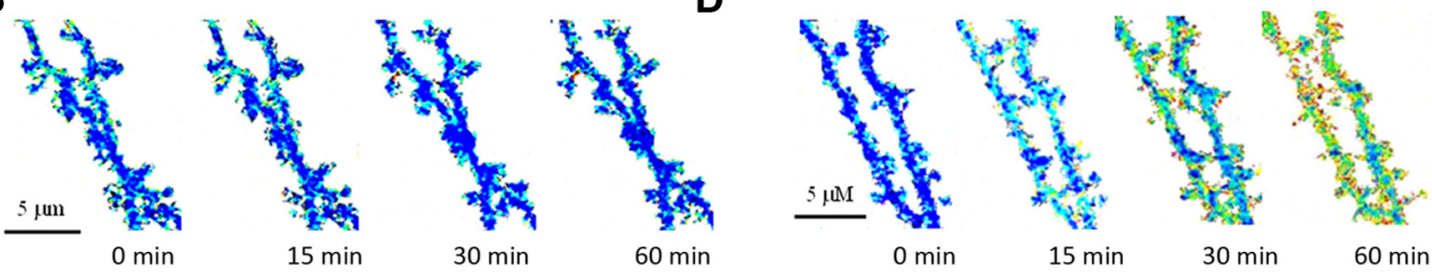

Figure 4. FRET assay from neurons expressing GFP-CaM and RFP-CaNA reports a rapid interaction of CaNA and CaM in dendritic spines induced by A $\beta$-containing TgCM. Cultured primary neurons at 9 DIV were transfected with GFP-CaM and RFP-CaN. Two weeks after transfection, neurons were incubated with either WtCM $(A, B)$ or $\operatorname{TgCM}(C, D)$ for the indicated times. The pseudocolored ratio images show that CaN is activated in live neurons as soon as $15 \mathrm{~min}$ after exposure to $A \beta$ in the whole cells and in dendritic spines $(\boldsymbol{C}, \boldsymbol{D})$. No change is detected when neurons were incubated with WtCM $(\boldsymbol{A}, \boldsymbol{B})$.

phore after stimulation with ionomycin. However, the GFP-CaM signal intensity remained stable when the RFP-CaNA fluorophore was not bleached.

We further confirmed the specificity of the interaction of CaNA-CaM or CaNA-CaNB in a native cellular environment by performing an immunolabeling FRET experiment (Knowles et al., 1999). Endogenous CaNA was immunolabeled with Alexa 488 as a donor fluorophore, and endogenous CaM was immunolabeled with $\mathrm{Cy} 3$ as an acceptor fluorophore. HEK cells treated with ionomycin showed a significantly higher 565/522 ratio compared with control (Fig. $1 E$ ). An alternative technique to measure FRET, FLIM, showed consistent results. In cells treated with ionomycin, the lifetime of Alexa 488-immunolabeled CaNA became significantly shorter compared with the lifetime in cells treated with DMSO, reflecting a transfer of energy between the donor and the acceptor (Fig. $1 F$ ). This technique relies only on the characteristics of the donor fluorophore, thus confirming that the results obtained with the ratiometric spectra approach reflects FRET interactions rather than spectral artifacts such as bleed through (Berezovska et al., 2003).

Together, the different FRET approaches used to detect interactions of CaNA-CaM demonstrate that change in FRET efficiency is sufficient to distinguish basal cellular CaN activity from stimulated states. The methods we used here report FRET signal consistently and are useful to monitor live-cell, real-time interactions of RFP-CaNA and GFP-CaM as well as interactions of the endogenous CaNA and CaM proteins, thus providing a great deal of flexibility in assay design.

CaN activation in an in vitro model of $\mathrm{AD}$ can be reported at a subcellular level by FRET assay

Previously, we have shown that, compared with wild-type cultured neurons, CaN activity measured by NFATc4 translocation to the nucleus is chronically elevated in neurons prepared from transgenic embryos expressing APP with the familial Swedish mutation (Tg2576 neurons) (Hsiao et al., 1996; Wu et al., 2010). This effect on NFAT nuclear localization could be blocked by immunodepletion of $\mathrm{A} \beta$ or treatment with $\mathrm{CaN}$ inhibitors (Wu et al., 2010). While these data support the idea that soluble oligomeric $\mathrm{A} \beta$ peptides induce $\mathrm{CaN}$ activation, they provide little spatial or temporal resolution. To test whether the FRET assay is sensitive enough to detect changes in interaction between CaNA-CaM or CaNA-CaNB under these circumstances, the FRET signal was compared between wild-type and Tg2576 neurons under basal conditions. In primary cultured neurons stained for endogenous CaNA-CaM or CaNA-CaNB, we observed a significant increase in the 565/522 ratio in cultured Tg2576 neurons compared with wild-type cultured neurons (Fig. 2). In wild-type neurons, the pseudocolored image, in which blue represents low FRET signal and red represents high FRET signal, shows only a very faint FRET signal (Fig. $2 A, E$ ). However, in accord with our previous observations of NFAT localization in the nucleus, Tg2576 cultured neurons show a strong FRET signal analyzed by both spectral FRET and FLIM-based FRET, indicating an interaction between CaNA and CaM (Fig. 2A-D). The spectral FRET assay indicates substantially increased interaction between CaNA-CaM, confirmed by similar data using the CaNA-CaNB pair in Tg2576 neurons (Fig. 2E,F). Moreover, the enhanced interaction between CaNA and CaM or CaNA and CaNB in Tg2576 neurons was distributed in the cytoplasm, nucleus, and both axon and dendritic processes, but the extent of activation was different in the three compartments. Quantitatively, there was a trend toward having a greater elevation of CaN activity in dendritic spines (1.2-fold for CaNA-CaM; 1.5 -fold for CaNA-CaNB; $n \geq 50$ neurons and $n \geq$ 200 spines in each condition; Fig. $2 B, F$ ) than in both the cytosol and nuclei (1.1-fold for CaNA-CaM; 1.3-fold for CaNA-CaNB). 


\section{A (CaNA-CaNB)}
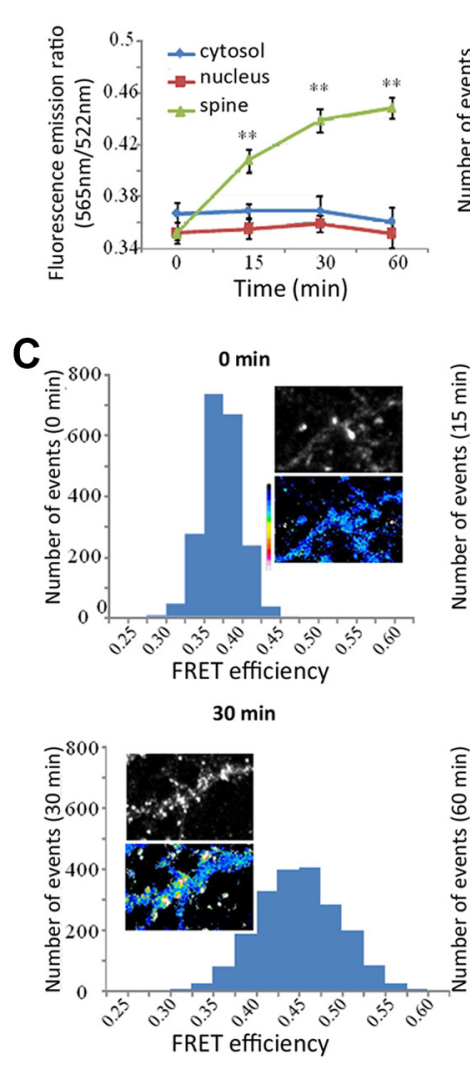

\section{B (CaNA-CaNB)}
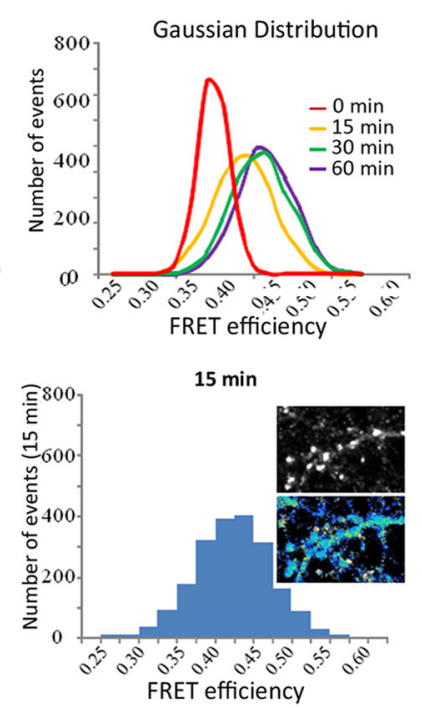

$60 \mathrm{~min}$

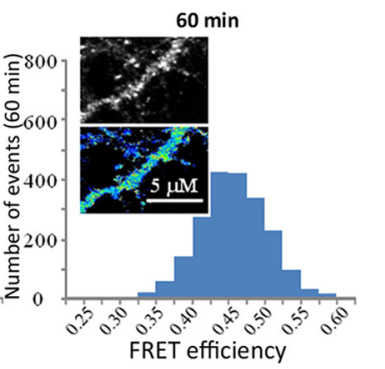

D (CaNA-CaM)

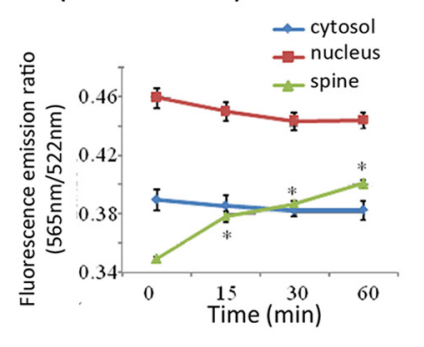

F
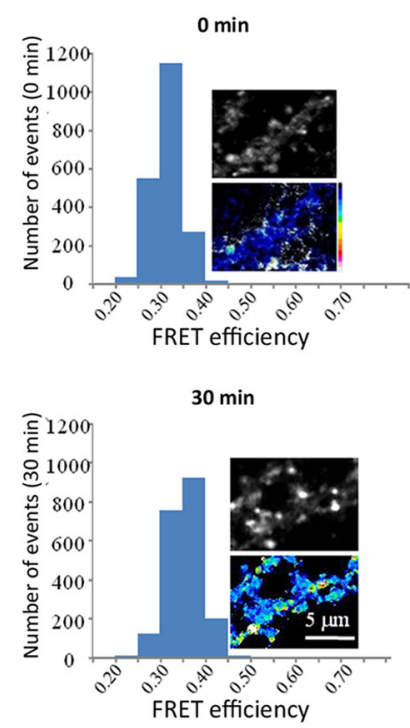

\section{E (CaNA-CaM)}
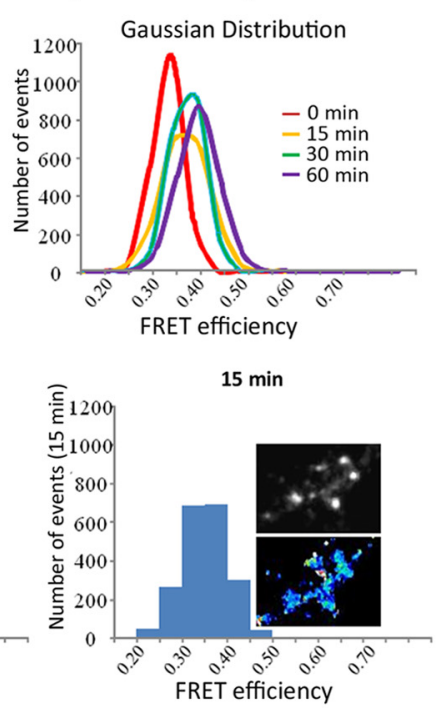

$60 \mathrm{~min}$

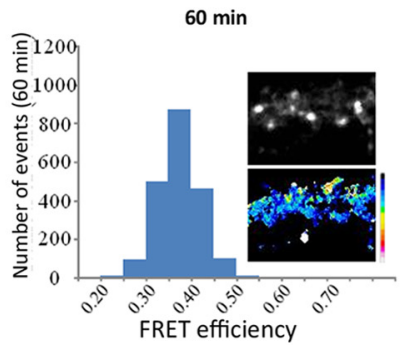

Figure 5. Time course of FRET changes in neurons treated with TgCM analyzed from endogenous CaNA/CaNB and CaNA/CaM FRET pairs. Cultured wild-type primary neurons at 24 DIV were treated with $\mathrm{TgCM}$, and then fixed with 4\% paraformaldehyde at indicated time points and immunolabeled with endogenous CaNA-Alexa 488 and either CaNB-Cy3 or CaM-Cy3.A, Time-dependent changes of the FRET based on interaction between endogenous CaNA and CaNB. Within 60 min, application of TgCM caused no significant difference in FRET levels of CaNA and CaNB in cytosolic and nuclear compartments ( $n \geq 45$ cells). However, significantly increased FRET signal between endogenous CaNA and CaNB in spines was detected as early as 15 min after TgCM application and increased with time. ${ }^{* *} p<0.01$. B, Gaussian distributions show FRET levels of endogenous CaNA and CaNB in spines in resting condition and changes with time in response to TgCM. C, Histograms of FRET values of CaNA and CaNB in response to $\mathrm{TgCM}$ application at indicated time points. In resting condition ( $0 \mathrm{~min}$ ), the FRET distribution in spine is dominated by a low FRET peak ( 0.36 ; top left panel). The FRET distribution was changed from basal to high level with time after application of TgCM. The FRET peak was 0.45 at 15 min (top right panel), 0.48 at 30 min (bottom left panel), and 0.48 at 60 min after $\mathrm{TgCM}$ application (bottom right panel). D, Similarly, time-dependent changes of the normalized FRET levels between CaN and CaM show an early increase in spines after 15 min, whereas no difference was observed in both the cytoplasmic and nuclear compartments up to $60 \mathrm{~min}$. $\boldsymbol{E}$, Gaussian distribution shows CaNA/CaM FRET levels in spines in resting condition and changes with time in response to $\mathrm{TgCM}$. F, Histograms of CaNA/CaM FRET values after $15 \mathrm{~min}$ (peak at 0.38; top right panel), 30 min (peak at 0.39; bottom left panel), and 60 min (peak at 0.4 ; bottom right panel) of $\mathrm{TgCM}$ exposure. In both $\boldsymbol{C}$ and $\boldsymbol{F}$, inset top panels show CaNA-Alexa 488 fluorescent in dendrites and spines and bottom panels show FRET images of dendritic spines. $n=200$ spines for each indicated time point; ${ }^{*} p<0.05$. Data represent mean \pm SEM.

Differential kinetics of CaN activation in dendritic spines and soma after applying conditioned media from Tg2576 neurons We recently reported that exposure of wild-type neurons for $24 \mathrm{~h}$ to $\mathrm{A} \beta$-containing conditioned media prepared from Tg2576 culture is capable of inducing $\mathrm{CaN}$ activation using NFAT nuclear translocation as a readout of CaN activation (Wu et al., 2010). NFAT translocation could be blocked by immunodepletion of the media with anti-A $\beta$ antibody or by blocking CaN with the pharmacological inhibitor $(3 S, 4 R, 5 S, 8 R$, $9 E, 12 S, 14 S, 15 R, 16 S, 18 R, 19 R, 26 a S)-5,6,8,11,12,13,14,15,16,17,18,19$, $24,25,26,26 a$-hexadecahydro-5,19-dihydroxy-3-[(1E)-2-[(1R,3R, $4 R$ )-4-hydroxy-3-methoxycyclohexyl]-1-methylethenyl]-14,16dimethoxy-4,10,12,18-tetramethyl-8-(2-propen-1-yl)-15,19epoxy-3H-pyrido[2,1-c] [1,4] oxaazacyclotricosine-1,7,20,21(4H, $23 H$ )tetrone (FK506). To characterize the precise spatial and temporal patterns of $\mathrm{CaN}$ activation, wild-type cultured neurons were exposed to $\mathrm{A} \beta$-containing conditioned media prepared from Tg2576 neurons. Consistent with our previous finding, application of $\mathrm{A} \beta$-containing conditioned media onto wild-type neurons for $24 \mathrm{~h}$ caused a marked increase in the 565/522 ratio of both CaNA-CaM and CaNA-CaNB (Fig.
$3 A, B)$, but no changes were observed in neurons exposed to conditioned media prepared from wild-type cultures. Importantly, significantly enhanced 565/522 ratio was detected in the nucleus, cytoplasm, and dendritic spines. This finding shows that application of $\mathrm{A} \beta$-containing TgCM causes $\mathrm{CaN}$ activation in all neuron compartments. This activation of $\mathrm{CaN}$ was directly related to the presence of $\mathrm{A} \beta$, as immunodepletion of $\mathrm{A} \beta$ from $\mathrm{Tg} 2576$ conditioned media with 3D6, a specific antibody to $A \beta$, prevented the increase in the $565 / 522$ ratio of both CaNA-CaM and CaNA-CaNB (Fig. $3 A, B$ ).

We next compared the spatiotemporal dynamics of $\mathrm{CaN}$ activation during $\mathrm{A} \beta$ exposure in living neurons expressing GFP$\mathrm{CaM}$ and RFP-CaNA. Figure $4 C$ shows pseudocolor images of a wild-type neuron expressing GFP-CaM and RFP-CaNA before $(0$ $\mathrm{min}$ ) and at 15, 30, and $60 \mathrm{~min}$ after application of TgCM. Exposure to TgCM triggered a rapid increase in the interactions between GFP-CaM and RFP-CaN in dendritic spines (Fig. 4D), whereas changes in the cytosolic and nuclear compartments were not detected for at least $60 \mathrm{~min}$ (Fig. 4C). Importantly, no change in FRET signal was observed after exposure to conditioned media from wild-type cultures (Fig. $4 A, B$ ). 
The rapid $\mathrm{CaN}$ activation in spines in response to TgCM application was further verified by using endogenous CaNAAlexa 488 and CaNB-Cy3 or CaN-Alexa 488 and CaM-Cy3 as FRET pairs. Significant increases in FRET between endogenous CaNA and CaNB or CaNA and CaM in dendritic spines were observed as early as $15 \mathrm{~min}$ after TgCM application (the earliest point examined) and reached their peak after $\sim 30$ min (Fig. $5 A, B, D, E$ ). The level of CaNA-CaNB or CaNA-CaM interaction persisted for up to $60 \mathrm{~min}$ with no sign of decline. Increases in the interaction of CaNA-CaM or CaNA-CaNB in spines indicate that a rapid and sustained CaNA activation was induced by TgCM. Comparing histograms of high FRET events among different time points for experiments across many spines revealed that FRET efficiency gradually and stably increased over a period of $0-60 \mathrm{~min}$ in most spine populations (Fig. 5C,F). High FRET observed in spines from CaNA$\mathrm{CaM}$ or CaNA-CaNB within minutes of TgCM exposure indicates a rapid $\mathrm{CaN}$ activation in response to acute $\mathrm{A} \beta$ stimulation. The intensity of the 565/522 ratio of both CaNA-CaM and CaNA-CaNB in the cytoplasm and nucleus was unchanged at 60 min after $\mathrm{A} \beta$ application but significantly increased at $6 \mathrm{~h}$ (FRET ratio increase for the CaNA-CaM pair: $\mathrm{cy}-$ toplasm, $0.53 \pm 0.017$; nucleus, $0.50 \pm 0.014$; FRET ratio increase for the CaNA-CaNB pair: cytoplasm, $0.51 \pm 0.008$; nucleus, $0.49 \pm 0.006 ; p<0.001$ compared with correspondent baseline; Fig. $6 A, B)$.

Because the role of $\mathrm{CaN}$ in neurons involves both structural and transcription factor targets, the subcellularly specialized pattern of $\mathrm{CaN}$ activation in response to $\mathrm{A} \beta$-containing $\mathrm{TgCM}$ stimulation may suggest that local posttranslational effects regulated by $\mathrm{CaN}$ in the spine occur essentially immediately, whereas CaNmediated transcriptional effects in the nucleus may be a delayed phenomenon.

To further dissect early and late events, CaN activation was measured by FRET in neurons exposed to $A \beta$-containing conditioned media for $60 \mathrm{~min}$, a period that induces significant $\mathrm{CaN}$ activation in the spine, and then washed off, and neurons were recovered in their original media for another $24 \mathrm{~h}$. As expected, neurons maintained in $\mathrm{A} \beta$-containing conditioned media for the entire $24 \mathrm{~h}$ had significantly increased FRET ratios of both CaNA$\mathrm{CaM}$ and CaNA-CaNB pairs (Fig. 6C,D). However, neurons exposed to $A \beta$-containing conditioned media for $60 \mathrm{~min}$, following washing off and incubation for $24 \mathrm{~h}$ in original conditioned media, showed basal levels of FRET signal in all three compartments. Thus, the $\mathrm{CaN}$ activation induced by $\mathrm{A} \beta$ is reversible if $\mathrm{A} \beta$ is removed after relatively brief exposure, and the progressive activation of $\mathrm{CaN}$ in the cytoplasm and nucleus can be aborted.

\section{Morphological consequences of rapid $\mathrm{CaN}$ activation in spines after applying $\mathrm{A} \boldsymbol{\beta}$-containing TgCM}

$\mathrm{CaN}$ function in neurons has been implicated in various aspects of synaptic plasticity, including regulation of dendritic spine
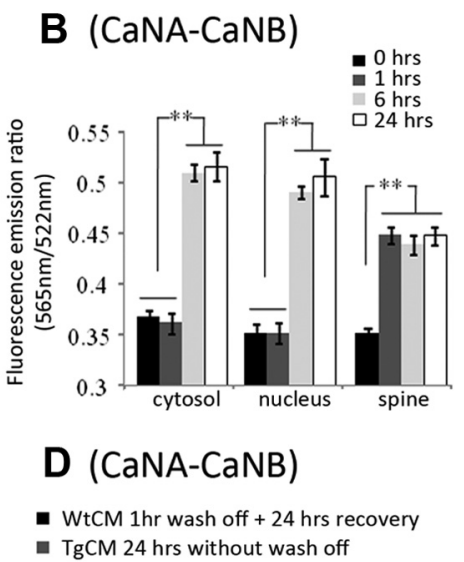

- TgCM $1 \mathrm{hr}$ wash off $+24 \mathrm{hrs}$ recovery

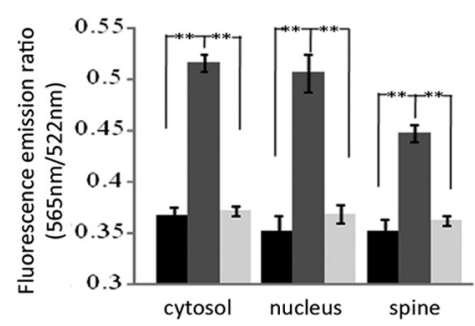

Figure 6. Later and sustained CaN activation in response to $\mathrm{TgCM}$ application detected by both endogenous CaNA-CaM and CaN-CaNB FRET pairs. $\boldsymbol{A}, \boldsymbol{B}$, Quantitative analysis of normalized spectral ratio (intensity ratio of $565-522 \mathrm{~nm}$ ) from different cellular compartments in wild-type neurons treated with $\mathrm{TgCM}$ at indicated time points. Significant increases in FRET signal between versible interaction of both CaNA-CaM $(\boldsymbol{C})$ and $\mathrm{CaN}-\mathrm{CaNB}(\boldsymbol{D})$ is induced by a brief exposure of neurons with $\mathrm{TgCM}$. A recovery of FRET signals to basal levels is observed when neurons were exposed to $A \beta$-containing medium for $1 \mathrm{~h}$ and washed out for $24 \mathrm{~h}$. CaN activation appear to be reversible. $n \geq 45$ cells and $n \geq 200$ spines; $^{*} p<0.05$; ${ }^{* *} p<0.001$. Data represent mean \pm SEM.

morphology (Halpain et al., 1998; Zhou et al., 2004; Wu et al., 2010). We previously observed that application of TgCM for $24 \mathrm{~h}$ decreases spine density in cultured neurons (Wu et al., 2010). To examine whether the rapid $\mathrm{CaN}$ activation in spines leads to spine loss or change in morphology, we compared the density of mature spines and of filopodia-like protrusions (evaluated independently) in mature cultured cortical neurons (21 DIV) $60 \mathrm{~min}$ after treatment with WtCM or TgCM. Quantitative analysis indicated that spine density in neurons treated with TgCM for 60 min was comparable with neurons treated with wild-type CM (Fig. $7 A, B$ ). In contrast to the relatively normal spine density, spine morphology was noticeably altered in neurons treated with TgCM (Fig. 7A,C). Neurons treated with wild-type CM for 60 min had the majority of spines with mushroom shape similar to those spines before treatment. Treatment with TgCM for $60 \mathrm{~min}$ led to a significant increase in the density of filopodia-like protrusions (Fig. 7C). The average number of filopodia-like protrusions versus total spines was $11 \%$ in neurons (21 DIV) before TgCM application, but dramatically increased to $24 \%$ at $15 \mathrm{~min}$, $32 \%$ at $30 \mathrm{~min}$, and $45 \%$ at $60 \mathrm{~min}$ after TgCM treatment, respectively (Fig. 7D). Immunodepletion of $\mathrm{A} \beta$ from TgCM with 3D6 or inhibition of $\mathrm{CaN}$ with its specific inhibitor FK506 markedly reduced the increase in number of filopodia-like protrusions (Fig. 7C), demonstrating that the effect of $\operatorname{Tg} 2576$ conditioned media is mediated by $\mathrm{A} \beta$ and consequent to activation of $\mathrm{CaN}$. Treatment with VIVIT, a specific inhibitor for NFAT activation, had no effects on the increase in number of filopodia-like protrusions, suggesting that CaN-mediated NFAT activation does not participate in the acute spine morphological alteration [unlike the effective protection from spine loss at $24 \mathrm{~h}$ due to NFAT 


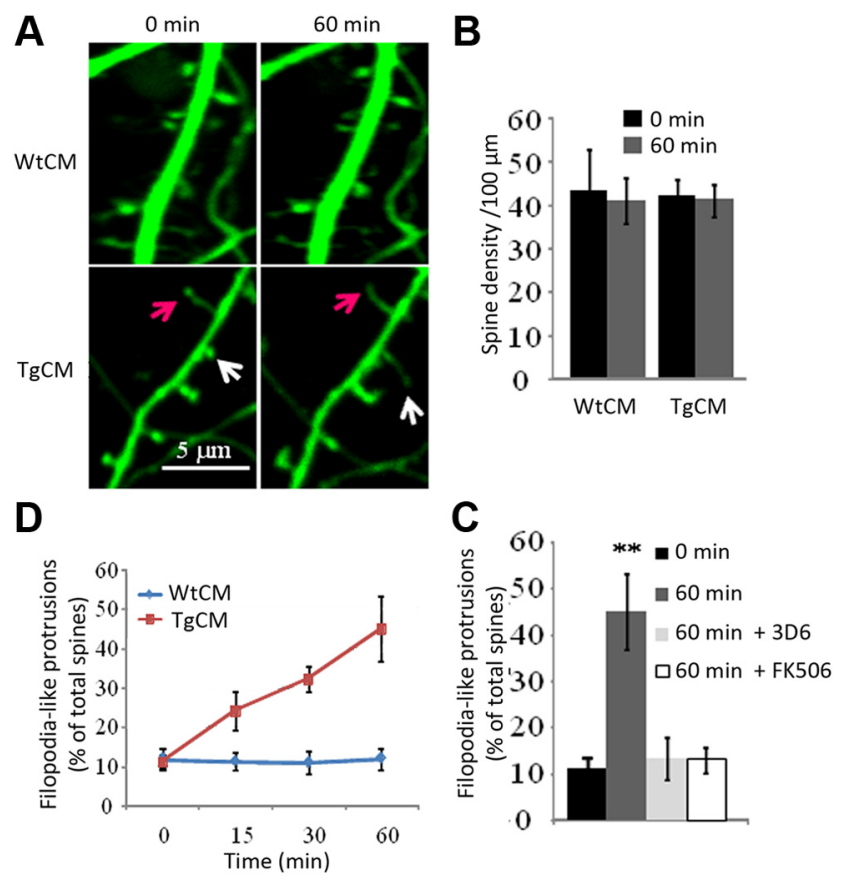

Figure 7. Changes in dendritic spine morphology and decreases in spine F-actin and synaptic surface GluR1 subunit of AMPA receptors occur during rapid CaN activation induced by $A \beta$ containing $\mathrm{TgCM}$. A, Exposure of wild-type neurons to WtCM or $\mathrm{TgCM}$ showed that treatment with $\mathrm{TgCM}$ induced a shrinkage of existing spines, as seen by spine length elongation (white arrows) and spine head disappearance (red arrows). $\boldsymbol{B}, \boldsymbol{C}$, Quantification of spine density ( $\boldsymbol{B}$ ) and filopodia-like protrusions (percentage of total spines) (C). $A \beta$ exposure for a period of $60 \mathrm{~min}$ caused spine length elongation and induced more filopodia-like protrusions without changing spine density. These responses were completely inhibited when $\mathrm{TgCM}$ was immunodepleted with $3 D 6$ or when cells were pretreated with $1 \mu \mathrm{m}$ FK506. D, The increase of filopodia-like protrusions was detected as soon as $15 \mathrm{~min}$ after incubation with $\mathrm{TgCM}$. Data are presented as mean \pm SEM. ${ }^{* *} p<0.001 ; n=$ total 200 spines in each condition, from three independent experiments.

blockade with VIVIT (Wu et al., 2010)]. This result suggests that, in mature neurons, $\mathrm{A} \beta$-containing $\mathrm{CM}$ from Tg2576 culture induces rapid $\mathrm{CaN}$ activation in spines, which causes acute spine morphological changes without affecting total spine density.

\section{Molecular consequences of rapid $\mathrm{CaN}$ activation in spines after applying $\mathrm{A} \boldsymbol{\beta}$-containing $\mathrm{TgCM}$}

Application of $\mathrm{A} \beta$-containing TgCM results in marked changes in spine morphology in cultured neurons that is blocked by inhibiting CaN activation (Fig. 7). As spine morphology and synaptic function are coordinated (Lippman and Dunaevsky, 2005; Dickstein et al., 2007; Bourne and Harris, 2008), we next examined whether $\mathrm{A} \beta$-containing conditioned media-induced $\mathrm{CaN}$ activation in spines also regulates glutamate receptors at synapses. To address this question, we labeled the surface GluR1 subunit of the AMPA receptor, which is a major mechanism contributing to synaptic plasticity, a key process for learning, memory, and other cognitive functions of the nervous system (Aramburu et al., 2000; Malenka and Bear, 2004). AMPA receptors are linked to F-actin by scaffold proteins, and actin depolymerization triggers loss of AMPA receptors (Allison et al., 2000; Shen et al., 2000; Zhou et al., 2001; Gomez et al., 2002). In cultured neurons, CaN activation induced by $\mathrm{A} \beta$-containing Tg2576 conditioned media or overexpression of a constitutively active CaN could lead to dephosphorylation of cofilin, a protein that triggers depolymerization of F-actin (Bailly and Jones, 2003) and regulates spine shape (Halpain et al., 1998). Consistent with this idea, in neurons treated with $\mathrm{A} \beta$-containing conditioned media for $60 \mathrm{~min}$, we noticed that the intensity ratio of phalloidin-stained F-actin in the spine to the dendritic shaft decreased markedly compared with neurons treated with wild-type conditioned media (spine/shaft ratio, wtCM, $3.20 \pm 0.12$; TgCM, $1.62 \pm 0.06 ; p<0.001$; Fig. $8 A, B)$. The significant decrease in spine/shaft fluorescence ratio is indicative of spine shrinkage and actin depolymerization (Gomez et al., 2002), which is in accord with our observations in GFP-expressing neurons (Fig. 7A). Immunodepletion of $\mathrm{A} \beta$ or inhibition of $\mathrm{CaN}$ prevented the ratio reduction (spine/shaft ratio, 3D6, $2.96 \pm 0.11$; FK506, $3.48 \pm$ 0.13 ), suggesting that $\mathrm{CaN}$ activation induced by $\mathrm{A} \beta$ leads to F-actin reorganization, which causes a change in spine morphology (Halpain et al., 1998; Bailly and Jones, 2003).

To determine whether the rapid $\mathrm{CaN}$ activation alters the GluR1 subunit of AMPA receptor content in spines, we measured the mean intensity of GluR1 immunofluorescence signal in individual spines by staining fixed, nonpermeabilized cultured neurons with an antibody against an extracellular epitope of the GluR1 AMPA receptor subunit. In neurons treated with wildtype conditioned media, line scan fluorescence intensity profiles through dendritic spines and adjacent regions of the dendritic shaft revealed the greatest enrichment of surface GluR1 in a punctuated pattern mainly distributed around dendritic spines with much lower levels present in the dendritic shafts, suggesting that the majority of surface GluR1 occurs in a synaptic spine localization. Following application of $\mathrm{A} \beta$-containing conditioned media for $60 \mathrm{~min}$, the amount of the punctate GluR1 around dendritic spines was dramatically reduced, and increased in the dendritic shaft. Quantification of fluorescence mean intensity ratios for GluR1 puncta around dendritic spines versus the dendritic shaft confirmed enrichment of GluR1 in synaptic spines in neurons treated with wild-type conditioned media, and the ratio was decreased dramatically in neurons treated with $\mathrm{A} \beta$ containing conditioned media for $60 \mathrm{~min}$ (spine/shaft ratio, wtCM, $1.42 \pm 0.15$; TgCM, $0.62 \pm 0.07 ; p<0.001$; Fig. $8 A, B$ ). These effects were specifically dependent on $\mathrm{A} \beta$ exposureinduced $\mathrm{CaN}$ activation; removal of $\mathrm{A} \beta$ from conditioned media with 3D6 immunodepletion or pretreating neurons with the CaN inhibitor FK506 prevented the Tg2576 conditioned mediainduced ratio decreases (spine/shaft ratio, 3D6, $1.35 \pm 0.09$; FK506, $1.41 \pm 0.08$ ). Thus, rapid activation of $\mathrm{CaN}$ in dendritic spines by $\mathrm{A} \beta$-containing conditioned media strongly reduces surface GluR1 and removes GluR1-containing AMPA receptors from synapses, potentially leading to weaker synaptic connections. Overall, these results indicate that $\mathrm{A} \beta$-containing conditioned media activates $\mathrm{CaN}$ rapidly in the spine, which causes similarly rapid changes in the synapse both structurally (F-actin reorganization) and biochemically (surface GluR1 reduction).

\section{Soluble $\mathrm{A} \boldsymbol{\beta}$ oligomers isolated by size exclusion}

chromatography from human postmortem AD brain induce rapid $\mathrm{CaN}$ activation in spines with consequent alteration of spine morphology and reduction of synaptic surface GluR1

To further characterize the specific role of $\mathrm{A} \beta$ oligomers on $\mathrm{CaN}$ mediated changes in spines, we next asked whether soluble $\mathrm{A} \beta$ isolated directly from human $\mathrm{AD}$ cortex activates $\mathrm{CaN}$ in spines. Nondenaturing SEC was used to isolate various $\mathrm{A} \beta$ species from brain homogenates of human control or AD. Both ELISA and Western blot analysis showed that SEC fractions 18-19th $\mathrm{ml}$ of $\mathrm{AD}$ brain, but not the same fractions of control brain, were markedly enriched in oligomeric forms of $\mathrm{A} \beta 40$ and $\mathrm{A} \beta 42$ (Wu et al., $2010)$. Application of oligomeric A $\beta$-enriched SEC fractions $18-$ 
A

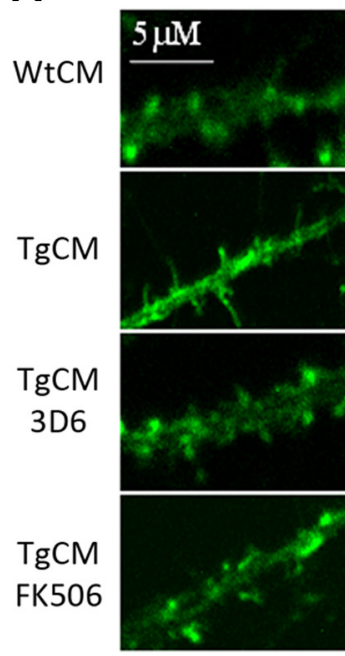

GluR1

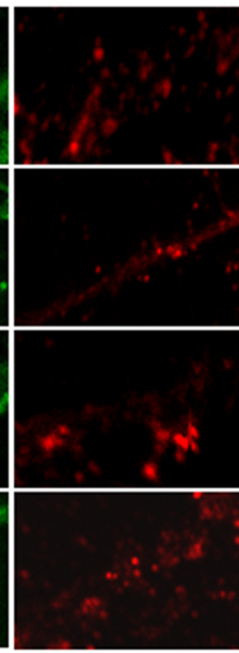

Merge

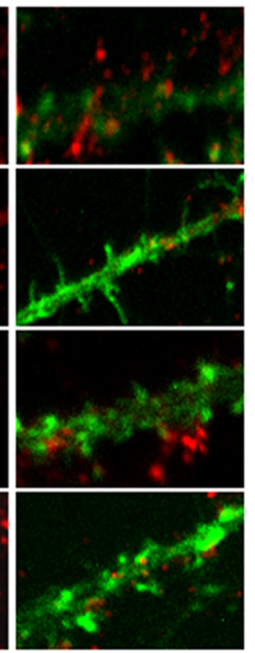

B

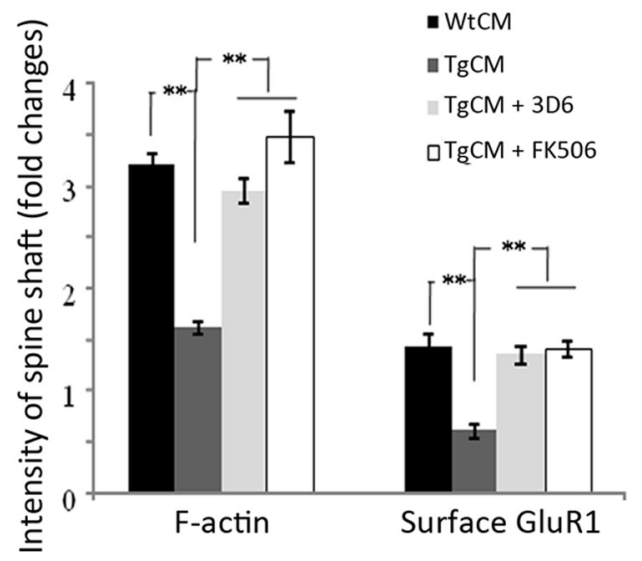

Figure 8. Exposure of cortical neurons to $A \beta$-containing medium leads to a reduction of GluR1 intensity at the surface of the dendritic spines. $A$, Dendrites from neurons treated with wild-type or TgCM for 60 min with or without 3D6 immunodepletion or FK506, and then stained with an antibody to the extracellular N-terminal domain of GluR1 to label surface AMPA receptors, and phalloidin to visualize F-actin. $B$, Quantification of the effects of oligomeric $A \beta$ on F-actin and surface GluR1 signal intensity (represented as ratios of spines vs shaft). Exposure of neurons to $A \beta$-containing $\mathrm{TgCM}$ reduces the ratios of both phalloidin-stained F-actin and surface GluR1 on the spine to the dendritic shafts, indicating a loss of F-actin and synaptic GluR1 from dendritic spines. This effect is blocked by $3 \mathrm{D6}$ and FK506. Data are presented as mean \pm SEM. ${ }^{* *} p<0.001 ; n=$ total 200 spines in each condition, from three independent experiments.

19th prepared from $\mathrm{AD}$ brain samples onto wild-type neurons for 60 min caused a rapid significant increase in the 565/522 ratio of both CaNA-CaM and CaNA-CaNB in dendritic spines with no measurable changes in the soma including cytoplasm and nucleus (for the CaNA-CaM pair: cytosol, control, $0.372 \pm$ 0.005 ; nuclei, $0.468 \pm 0.011$; spine, $0.353 \pm 0.004 ; \mathrm{AD}$, cytosol, $0.385 \pm 0.002$; nuclear, $0.467 \pm 0.013$; spine, $0.383 \pm 0.005 ; p<$ 0.001 , control spine vs $\mathrm{AD}$ spine; for the CaNA-CaNB pair: control cytosol, $0.374 \pm 0.007$; nuclei, $0.376 \pm 0.004$; spine, $0.364 \pm$ 0.007 ; AD, cytosol, $0.369 \pm 0.009$; nuclei, $0.371 \pm 0.005$; spine, $0.451 \pm 0.017 ; p<0.001$, control spine vs AD spine; Fig. $9 A, B)$. In contrast, wild-type neurons exposed to the same SEC fractions prepared from control brain samples showed low FRET efficiency. Immunodepletion of $A \beta$ from fractions 18-19th of $A D$ samples with 3D6 prevented the increase in the 565/522 ratio of both CaNA-CaM and CaNA-CaNB (for the CaNA-CaM pair: $\mathrm{AD}$ plus $3 \mathrm{D} 6,0.349 \pm 0.003 ; p<0.001, \mathrm{AD}$ spine vs $\mathrm{AD}$ plus $3 \mathrm{D} 6$ spine; for the CaNA-CaNB pair: $0.374 \pm 0.117 ; p=0.002$, AD spine vs $\mathrm{AD}$ plus $3 \mathrm{D} 6$; Fig. $9 A, B$ ). This result provides a direct demonstration that $\mathrm{A} \beta$ oligomers from the human $\mathrm{AD}$ brain induce rapid $\mathrm{CaN}$ activation in spines.

With the phalloidin-stained F-actin fluorescence signal, we observed that neurons treated with $\mathrm{A} \beta$-enriched SEC fractions prepared from $\mathrm{AD}$ brain samples for $60 \mathrm{~min}$ appeared to have more filopodia-like outgrowths, and the density of filopodia-like protrusions was significantly increased. The average number of filopodia-like protrusions versus total spines was $46 \%$ in neurons treated with fractions of AD brain samples compared with $12 \%$ in neurons treated with the same fraction of control brain samples (Fig. 9C). This morphological phenotype in neurons exposed to $\mathrm{A} \beta$-enriched SEC fractions of AD brain samples was also confirmed by measuring the fluorescence mean intensity ratios of F-actin for spine versus dendritic shaft. The ratios were significantly reduced in neurons treated with AD SEC fractions compared with neurons treated with the same SEC fractions prepared from control brain samples (spine/shaft ratio, control, $2.39 \pm$ $0.09 ; \mathrm{AD}, 1.67 \pm 0.07 ; p<0.001$; Fig. $9 E, F)$. In agreement with data from Figure $7 B$, incubation of neurons with SEC fractions from both control and $\mathrm{AD}$ brain samples for only $60 \mathrm{~min}$ did not affect total spine density (Fig. 9D). SEC fractions from AD brain samples were incapable of inducing spine morphological changes if these SEC fractions were immunodepleted with 3D6 (percentage of filopodia-like protrusions, $12.5 \pm 2.7 \%$; spine/shaft ratio, $2.22 \pm 0.07$; Fig. $9 C, F)$, demonstrating that $\mathrm{A} \beta$ is required for the structural modification in spines. Moreover, inhibition of $\mathrm{CaN}$ with its specific inhibitor FK506 markedly reduced the increase in number of filopodia-like protrusions and significantly prevented the decreased ratios of F-actin (percentage of filopodia-like protrusions, $13.7 \pm 2.8 \%$; spine/shaft ratio, $2.23 \pm 0.11$; Fig. $9 C, F$ ). This result suggests that $\mathrm{A} \beta$ isolated from $\mathrm{AD}$ brain induces rapid $\mathrm{CaN}$ activation in spines, which causes acute spine morphological changes without immediately affecting spine density.

We also compared levels of surface GluR1 in neurons treated with SEC fractions from both control and AD brain samples. Neurons treated with $\mathrm{A} \beta$-enriched SEC fractions prepared from $\mathrm{AD}$ brain samples for 60 min showed decreases in mean surface GluR1 fluorescence in dendritic spine compared neurons exposed to the same SEC fractions prepared from control brain samples (spine/shaft ratio, control, $1.33 \pm 0.13 ; \mathrm{AD}, 0.88 \pm 0.04$; $p<0.001$; Fig. $9 E, F)$. This effect was caused by A $\beta$-induced local CaN activation as immunodepletion of $A \beta$ from AD SEC samples or coapplication of FK506 with AD SEC samples blocked the change in surface levels of GluR1 in synaptic sites (spine/shaft ratio, $\mathrm{AD}$ plus $3 \mathrm{D} 6,1.30 \pm 0.12$; AD plus FK506, $1.21 \pm 0.13$; Fig. $9 E, F)$. Overall, this observation provides further evidence for the involvement of local $\mathrm{CaN}$ activation by toxic $\mathrm{A} \beta$ species in $\mathrm{AD}$ pathogenesis.

\section{Discussion}

We describe a spectral FRET-based method to quantitatively measure CaN activation dynamics at a subcellular level in cultured neurons. The spectral FRET-based assay defines two localization states of $\mathrm{CaN}$ activation in response to soluble $\mathrm{A} \beta$ oligomer stimulation: first, a robust CaN activation is initiated in spines after a brief $\mathrm{A} \beta$ exposure; second, a delayed but sustained $\mathrm{CaN}$ activation is observed in the cytoplasm and the nucleus by 

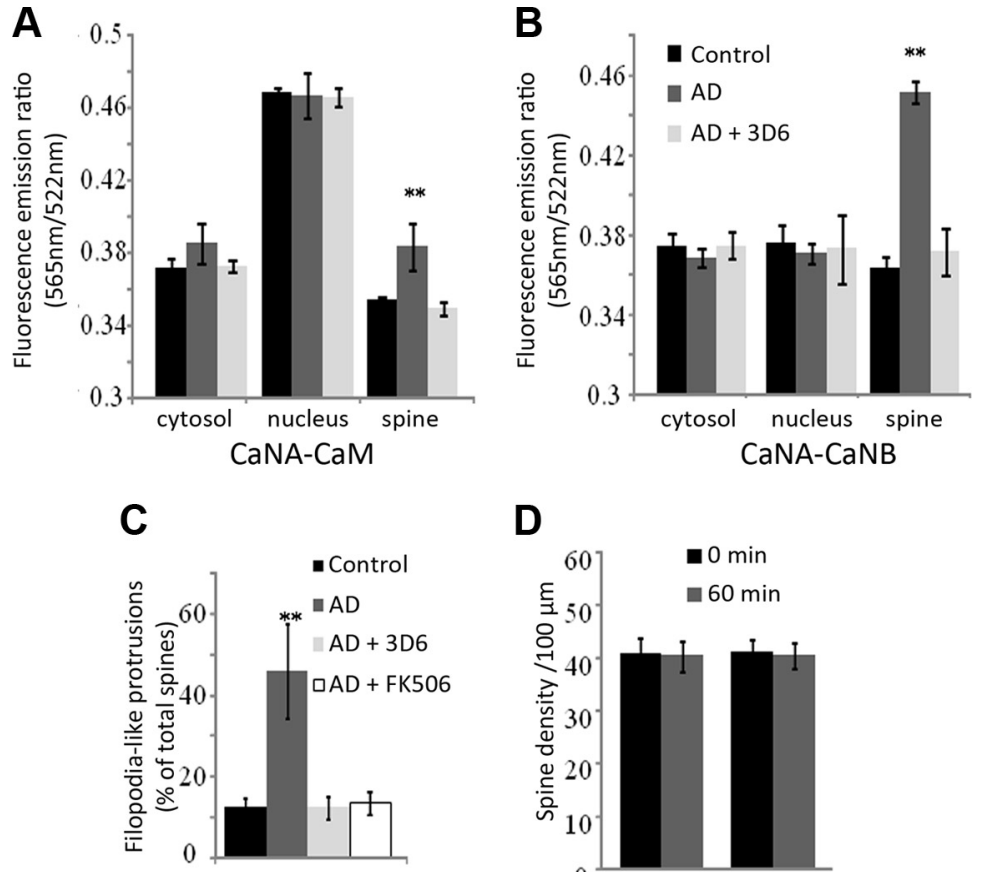

D

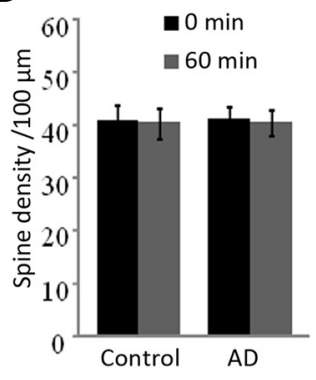

E

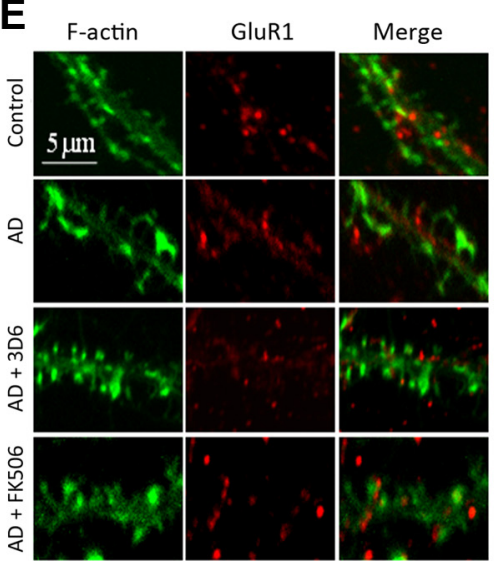

$\mathbf{F}$

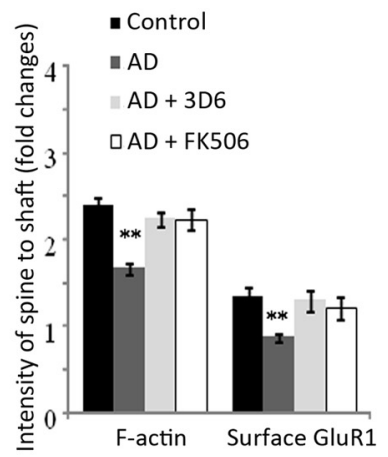

Figure 9. Soluble $A \beta$ oligomers isolated by SEC from human postmortem AD brain induces rapid CaN activation in spines, alters spine morphology, and reduces spine F-actin and synaptic surface GluR1. A, B, Quantitative analysis of normalized spectral ratio (intensity ratio of 565-522 nm) from different neuron compartments in neurons exposed to control or AD SEC fractions with or without 3D6 or FK506. Neurons treated with AD SEC fractions for 60 min show significantly higher FRET of both CaNA-CaM $(\boldsymbol{A})$ and CaNA-CaNB $(\boldsymbol{B})$ in spines compared with neurons treated with the comparable SEC fraction from a control brain, whereas FRET signal of CaNA-CaM or CaNA-CaNB in the cytosolic and nuclear compartments was not changed in neurons treated with either control or AD SEC fractions ( $n \geq 45$ cells and $n \geq 200$ spines; ${ }^{* *} p<0.001$ ). Data represent mean \pm SEM. C, Quantification of filopodia-like protrusions (percentage of total spines). AD SEC fraction exposure for a period of 60 min caused spine length elongation and induced more filopodia-like protrusions. This response was completely inhibited when cells were pretreated with $1 \mu \mathrm{M}$ FK506 or Tg2576-containing conditioned media was immunodepleted with 3D6. D, Exposure of neurons to oligomeric $A \beta$-enriched SEC fractions prepared from AD brain samples for 60 min caused no changes in spine density compared with that of neurons exposed to similar SEC fractions prepared from control brain. $\boldsymbol{E}, \boldsymbol{F}$, Decreases in spine F-actin and synaptic surface GluR1 subunit of AMPA receptors during rapid CaN activation induced by SEC-isolated A $\beta$ oligomers prepared from AD human brains. Neurons treated with AD SEC fraction for 60 min at indicated experimental conditions (with or without 3D6 immunodepletion or in the presence or absence of the (aN inhibitor FK506), and then stained with an antibody to the extracellular N-terminal domain of GluR1 to label surface AMPA receptors, and phalloidin to visualize F-actin $(\boldsymbol{E})$. Exposure of neurons to SEC-isolated A $\beta$ oligomers prepared from $A D$ human brains reduces the ratios of both phalloidin-stained F-actin and surface GluR1 on the spine to the dendritic shafts, indicating a loss of F-actin and synaptic GluR1 from dendritic spines. This effect was blocked by 3D6 and FK506 (F). $n=$ total 200 spines in each condition, from three independent experiments. ${ }^{* *} p<0.001$. Data are presented as mean \pm SEM.

prolonged $\mathrm{A} \beta$ exposure. Acute activation of $\mathrm{CaN}$ in spines caused by $\mathrm{A} \beta$-containing conditioned media is sufficient to induce spine structural changes and affect levels of postsynaptic proteins including F-actin and GluR1-containing AMPA receptors. Notably, the disease-associated $\mathrm{A} \beta$ species, which are isolated by SEC from human $\mathrm{AD}$ brain, induces a rapid $\mathrm{CaN}$ activation specifically and locally in spines with consequent alteration in spine morphology and reduction in postsynaptic proteins. Our results provide the first demonstration that the temporal and spatial precision of $\mathrm{CaN}$ activation in response to $\mathrm{A} \beta$ stimulation involves compartmentalized modulation; the disease-associated $\mathrm{A} \beta$ oligomers initiate rapid, local posttranslational responses downstream of $\mathrm{CaN}$ in spines.

FRET-based assays have been extensively used as a method to image molecular processes in the natural environment such as protein-protein interaction of several signaling pathways, primarily taking advantage of overexpressed tagged proteins (Stryer, 1978; Selvin, 2000; Jares-Erijman and Jovin, 2003). We have extended this technology to $\mathrm{CaN}$ and have used different approaches to detect $\mathrm{CaN}$ activation in vitro for live-cell imaging using GFP-RFP tags and endogenous proteins after immunostaining. CaN activation is widely studied in the immune system and in numerous disease processes, including congestive heart failure (Clipstone and Crabtree, 1992; Crabtree and Schreiber, 2009). In the CNS, activation of CaN has been implicated in the context of normal learning and memory, as well as diseases including glaucoma, spinal cord trauma, kainate injury, and ischemia (Morioka et al., 1999; Springer et al., 2000; Wu et al., 2004; Huang et al., 2005). In the context of Alzheimer's disease, CaN was shown to be abnormally activated in neurons but also in glia, thus participating to neuroinflammatory processes that are tightly associated with the progression of the disease (Abdul et al., 2009). The tools we describe here may therefore prove useful in monitoring the role of $\mathrm{CaN}$ in different cell types, with improved spatial and temporal resolution.

The FRET-based tools described here allow us to specifically address questions such as the kinetics of CaNA/CaM and CaNA/ CaNB interactions following stimulation in neuronal cells and provides a tool for evaluating the kinetics of spine or nuclear activation of calcineurin after physiologic or pathologic stimuli. A previous attempt to monitor CaN activation using a phosphatase activity-dependent molecular switch was based on the N-terminal regulatory domain of the NFAT1 that were sandwiched between the enhanced cyan fluorescent protein (ECFP) and a circularly permuted version of the yellow fluorescent protein, Venus, as a specific substrate of CaN (Newman and Zhang, 2008). However, the overexpressed ECFP-Venus FRET pair has a limited response, is indirect in that it is restricted to report only CaN-mediated NFAT activation, is sensitive to NFAT phosphorylation events, and does not provide spatial in- 
formation about CaN activation. By contrast, the conformational changes induced by the interaction of CaNA-CaM or CaNA-CaNB directly report CaN activation toward all its substrates. This assay can also be adapted for transfected or endogenous protein assays and provides outstanding spatial and temporal resolution. As demonstrated by Stemmer and Klee (1994) in a previous study, the $K_{\mathrm{d}}$ of the $\mathrm{CaNB} \mathrm{Ca}{ }^{2+}$ binding sites is very high (10 $\left.\mathrm{nM}\right)$, so that CaNA and CaNB may be associated even at baseline. Recent pull-down/mass spectroscopy data suggest that the CaNA-CaNB interaction increases significantly with cellular stress (Kozubowski et al., 2011). From our data, we cannot distinguish whether CaNA and CaNB interact at baseline and then change their conformation when activated by calcium, or whether the association increases with an influx of calcium, or both; regardless, activation of calcineurin leads to a change in the conformation so that the fluorophores come close enough to one another to support FRET.

Our results indicate that $\mathrm{CaN}$ activity is significantly increased in spines over a period of $0-60$ min after $A \beta$ oligomer application. Although overt spine loss does not occur acutely, substrates located in the spine compartment are immediately targeted by $\mathrm{CaN}$ and synaptic function could be substantially affected during the early phase of CaN activation. For example, the early phase of $\mathrm{CaN}$ activation alters spine morphology and affects levels of surface GluR1-containing receptors in spines, confirming a strong local cellular consequence of $\mathrm{CaN}$ activation caused by acute $\mathrm{A} \beta$ oligomer stimulation (Hsieh et al., 2006). However, neurons have a late-phase $\mathrm{CaN}$ activation in soma that starts $6 \mathrm{~h}$ after $\mathrm{A} \beta$ oligomer application, lasts for at least $24 \mathrm{~h}$, and induces NFAT nuclear translocation (Wu et al., 2010). Neuron function could be potentially affected by both CaN-mediated posttranslational and transcriptional processes. For example, spine loss occurs in $24 \mathrm{~h}$ and can be blocked by VIVIT, which prevents NFAT transcriptional activation (Wu et al., 2010), whereas changes in spine morphology, which is associated with loss of postsynaptic proteins including F-actin and GluR1 subunit of AMPA receptors, occurs within the first hour and is not affected by VIVIT. Moreover, the FRET ratios of both CaNA-CaM and CaNA-CaNB obtained from all three compartments in neurons exposed to $\mathrm{A} \beta$ oligomers for $60 \mathrm{~min}$ followed by washing off and then returned to original conditioned media for $24 \mathrm{~h}$ showed only basal level CaN activation (Fig. 6C,D). This result indicates that the effect of the 60 min exposure to $\mathrm{A} \beta$ oligomers on $\mathrm{CaN}$ activation is temperate and reversible, and is a completely local event, which is not sufficient to extend its effect to the soma and initiate the latephase $\mathrm{CaN}$ activation. This also suggests that prolonged $\mathrm{A} \beta$ oligomer exposure is required for CaN activation in cytoplasm and nuclei. As altering $\mathrm{CaN}$ phosphatase activity is considered to be critical for physiological or pathological neuronal plasticity, the subcellular temporal properties of CaN activation in response to $\mathrm{A} \beta$ oligomer stimulation we observed here may be important to understand synaptic alterations during $\mathrm{AD}$ pathogenesis.

\section{References}

Abdul HM, Sama MA, Furman JL, Mathis DM, Beckett TL, Weidner AM, Patel ES, Baig I, Murphy MP, LeVine H 3rd, Kraner SD, Norris CM (2009) Cognitive decline in Alzheimer's disease is associated with selective changes in calcineurin/NFAT signaling. J Neurosci 29:12957-12969.

Allison DW, Chervin AS, Gelfand VI, Craig AM (2000) Postsynaptic scaffolds of excitatory and inhibitory synapses in hippocampal neurons: maintenance of core components independent of actin filaments and microtubules. J Neurosci 20:4545-4554.

Aramburu J, Rao A, Klee CB (2000) Calcineurin: from structure to function. Curr Top Cell Regul 36:237-295.

Bailly M, Jones GE (2003) Polarised migration: cofilin holds the front. Curr Biol 13:R128-R130.
Berezovska O, Ramdya P, Skoch J, Wolfe MS, Bacskai BJ, Hyman BT (2003) Amyloid precursor protein associates with a nicastrin-dependent docking site on the presenilin $1-\gamma$-secretase complex in cells demonstrated by fluorescence lifetime imaging. J Neurosci 23:4560-4566.

Berezovska O, Lleo A, Herl LD, Frosch MP, Stern EA, Bacskai BJ, Hyman BT (2005) Familial Alzheimer's disease presenilin 1 mutations cause alterations in the conformation of presenilin and interactions with amyloid precursor protein. J Neurosci 25:3009-3017.

Berridge MJ, Lipp P, Bootman MD (2000) The versatility and universality of calcium signalling. Nat Rev Mol Cell Biol 1:11-21.

Bittinger MA, McWhinnie E, Meltzer J, Iourgenko V, Latario B, Liu X, Chen $\mathrm{CH}$, Song C, Garza D, Labow M (2004) Activation of cAMP response element-mediated gene expression by regulated nuclear transport of TORC proteins. Curr Biol 14:2156-2161.

Blumenthal DK, Takio K, Hansen RS, Krebs EG (1986) Dephosphorylation of cAMP-dependent protein kinase regulatory subunit (type II) by calmodulin-dependent protein phosphatase. Determinants of substrate specificity. J Biol Chem 261:8140-8145.

Bourne JN, Harris KM (2008) Balancing structure and function at hippocampal dendritic spines. Annu Rev Neurosci 31:47-67.

Clipstone NA, Crabtree GR (1992) Identification of calcineurin as a key signalling enzyme in T-lymphocyte activation. Nature 357:695-697.

Crabtree GR, Schreiber SL (2009) SnapShot: $\mathrm{Ca}^{2+}$-calcineurin-NFAT signaling. Cell 138:210, 210.e1.

Dickstein DL, Kabaso D, Rocher AB, Luebke JI, Wearne SL, Hof PR (2007) Changes in the structural complexity of the aged brain. Aging Cell 6:275-284.

Enz A, Shapiro G, Chappuis A, Dattler A (1994) Nonradioactive assay for protein phosphatase $2 \mathrm{~B}$ (calcineurin) activity using a partial sequence of the subunit of cAMP-dependent protein kinase as substrate. Anal Biochem 216:147-153.

Gomez LL, Alam S, Smith KE, Horne E, Dell'Acqua ML (2002) Regulation of A-kinase anchoring protein 79/150-cAMP-dependent protein kinase postsynaptic targeting by NMDA receptor activation of calcineurin and remodeling of dendritic actin. J Neurosci 22:7027-7044.

Halpain S, Hipolito A, Saffer L (1998) Regulation of F-actin stability in dendritic spines by glutamate receptors and calcineurin. J Neurosci 18:9835-9844.

Hashimoto T, Wakabayashi T, Watanabe A, Kowa H, Hosoda R, Nakamura A, Kanazawa I, Arai T, Takio K, Mann DM, Iwatsubo T (2002) CLAC: a novel Alzheimer amyloid plaque component derived from a transmembrane precursor, CLAC-P/collagen type XXV. EMBO J 21:1524-1534.

Hsiao K, Chapman P, Nilsen S, Eckman C, Harigaya Y, Younkin S, Yang F, Cole G (1996) Correlative memory deficits, Abeta elevation, and amyloid plaques in transgenic mice. Science 274:99-102.

Hsieh H, Boehm J, Sato C, Iwatsubo T, Tomita T, Sisodia S, Malinow R (2006) AMPAR removal underlies Abeta-induced synaptic depression and dendritic spine loss. Neuron 52:831-843.

Huang W, Fileta JB, Dobberfuhl A, Filippopolous T, Guo Y, Kwon G, Grosskreutz CL (2005) Calcineurin cleavage is triggered by elevated intraocular pressure, and calcineurin inhibition blocks retinal ganglion cell death in experimental glaucoma. Proc Natl Acad Sci USA 102:12242-12247.

Jares-Erijman EA, Jovin TM (2003) FRET imaging. Nat Biotechnol 21:1387-1395.

Klee CB, Crouch TH, Krinks MH (1979) Calcineurin: a calcium- and calmodulin-binding protein of the nervous system. Proc Natl Acad Sci U S A 76:6270-6273.

Klee CB, Ren H, Wang X (1998) Regulation of the calmodulin-stimulated protein phosphatase, calcineurin. J Biol Chem 273:13367-13370.

Knowles RB, Wyart C, Buldyrev SV, Cruz L, Urbanc B, Hasselmo ME, Stanley HE, Hyman BT (1999) Plaque-induced neurite abnormalities: implications for disruption of neural networks in Alzheimer's disease. Proc Natl Acad Sci U S A 96:5274-5279.

Kozubowski L, Thompson JW, Cardenas ME, Moseley MA, Heitman J (2011) Association of calcineurin with the COPI protein Sec28 and the COPII protein Sec13 revealed by quantitative proteomics. PLoS One 6:e25280.

Lautermilch NJ, Spitzer NC (2000) Regulation of calcineurin by growth cone calcium waves controls neurite extension. J Neurosci 20:315-325.

Lippman J, Dunaevsky A (2005) Dendritic spine morphogenesis and plasticity. J Neurobiol 64:47-57. 
Liu G, Martins IH, Chiorini JA, Davidson BL (2005) Adeno-associated virus type 4 (AAV4) targets ependyma and astrocytes in the subventricular zone and RMS. Gene Ther 12:1503-1508.

Malenka RC, Bear MF (2004) LTP and LTD: an embarrassment of riches. Neuron 44:5-21.

Martin ZS, Neugebauer V, Dineley KT, Kayed R, Zhang W, Reese LC, Taglialatela G (2012) alpha-Synuclein oligomers oppose long-term potentiation and impair memory through a calcineurin-dependent mechanism: relevance to human synucleopathic diseases. J Neurochem 120:440-452.

Morioka M, Hamada J, Ushio Y, Miyamoto E (1999) Potential role of calcineurin for brain ischemia and traumatic injury. Prog Neurobiol 58:1-30.

Morishita W, Marie H, Malenka RC (2005) Distinct triggering and expression mechanisms underlie LTD of AMPA and NMDA synaptic responses. Nat Neurosci 8:1043-1050.

Newman RH, Zhang J (2008) Visualization of phosphatase activity in living cells with a FRET-based calcineurin activity sensor. Mol Biosyst 4:496-501.

Park KS, Mohapatra DP, Misonou H, Trimmer JS (2006) Graded regulation of the Kv2.1 potassium channel by variable phosphorylation. Science 313:976-979.

Schwartz N, Schohl A, Ruthazer ES (2009) Neural activity regulates synaptic properties and dendritic structure in vivo through calcineurin/NFAT signaling. Neuron 62:655-669.

Sellar KJ, van Rossum HH, Romijn FP, Smit NP, de Fijter JW, van Pelt J (2006) Spectrophotometric assay for calcineurin activity in leukocytes isolated from human blood. Anal Biochem 358:104-110.

Selvin PR (2000) The renaissance of fluorescence resonance energy transfer. Nat Struct Biol 7:730-734.

Shen L, Liang F, Walensky LD, Huganir RL (2000) Regulation of AMPA receptor GluR1 subunit surface expression by a $4.1 \mathrm{~N}$-linked actin cytoskeletal association. J Neurosci 20:7932-7940.

Springer JE, Azbill RD, Nottingham SA, Kennedy SE (2000) Calcineurinmediated $\mathrm{BAD}$ dephosphorylation activates the caspase- 3 apoptotic cascade in traumatic spinal cord injury. J Neurosci 20:7246-7251.

Stemmer PM, Klee CB (1994) Dual calcium ion regulation of calcineurin by calmodulin and calcineurin B. Biochemistry 33:6859-6866.
Stryer L (1978) Fluorescence energy transfer as a spectroscopic ruler. Annu Rev Biochem 47:819-846.

Tavalin SJ, Colledge M, Hell JW, Langeberg LK, Huganir RL, Scott JD (2002) Regulation of GluR1 by the A-kinase anchoring protein 79 (AKAP79) signaling complex shares properties with long-term depression. J Neurosci 22:3044-3051.

Townsend M, Shankar GM, Mehta T, Walsh DM, Selkoe DJ (2006) Effects of secreted oligomers of amyloid beta-protein on hippocampal synaptic plasticity: a potent role for trimers. J Physiol 572:477-492.

Uemura K, Lill CM, Li X, Peters JA, Ivanov A, Fan Z, DeStrooper B, Bacskai BJ, Hyman BT, Berezovska O (2009) Allosteric modulation of PS1/ gamma-secretase conformation correlates with amyloid beta(42/40) ratio. PLoS One 4:e7893.

Wang KK, Villalobo A, Roufogalis BD (1989) Calmodulin-binding proteins as calpain substrates. Biochem J 262:693-706.

Wang Y, Shibasaki F, Mizuno K (2005) Calcium signal-induced cofilin dephosphorylation is mediated by Slingshot via calcineurin. J Biol Chem 280:12683-12689.

Winder DG, Sweatt JD (2001) Roles of serine/threonine phosphatases in hippocampal synaptic plasticity. Nat Rev Neurosci 2:461-474.

Wu HY, Tomizawa K, Oda Y, Wei FY, Lu YF, Matsushita M, Li ST, Moriwaki A, Matsui H (2004) Critical role of calpain-mediated cleavage of calcineurin in excitotoxic neurodegeneration. J Biol Chem 279:4929-4940.

Wu HY, Hudry E, Hashimoto T, Kuchibhotla K, Rozkalne A, Fan Z, SpiresJones T, Xie H, Arbel-Ornath M, Grosskreutz CL, Bacskai BJ, Hyman BT (2010) Amyloid $\beta$ induces the morphological neurodegenerative triad of spine loss, dendritic simplification, and neuritic dystrophies through calcineurin activation. J Neurosci 30:2636-2649.

Zhou Q, Xiao M, Nicoll RA (2001) Contribution of cytoskeleton to the internalization of AMPA receptors. Proc Natl Acad Sci U S A 98:1261-1266.

Zhou Q, Homma KJ, Poo MM (2004) Shrinkage of dendritic spines associated with long-term depression of hippocampal synapses. Neuron 44: 749-757. 